The Astrophysical Journal, 685:181-193, 2008 September 20

(C) 2008. The American Astronomical Society. All rights reserved. Printed in U.S.A.

\title{
MOLECULAR GAS AND DUST IN ARP 94: THE FORMATION OF A RECYCLED GALAXY IN AN INTERACTING SYSTEM
}

\author{
U. LISENFELD \\ Departamento de Física Teórica y del Cosmos, Universidad de Granada, Spain and Instituto de Astrofísica de Andalucía, \\ CSIC, Apdo. 3004, 18080 Granada, Spain; ute@ugr.es \\ C. G. Mundell ${ }^{1}$ \\ Astrophysics Research Institute, Liverpool John Moores University, Twelve Quays House, Egerton Wharf, \\ Birkenhead CH41 1LD, UK; cgm@astro.livjm.ac.uk \\ E. SCHINNERER \\ Max-Planck-Institut für Astronomie, Königstuhl 17, 69117 Heidelberg, Germany; schinner@mpia-hd.mpg.de \\ P. N. Appleton \\ NASA Herschel Science Center (NHSC), California Institute of Technology, MC 100-22, \\ 1200 East California Boulevard, Pasadena, CA 91125; apple@iapc.caltech.edu \\ AND \\ J. Allsopp \\ Astrophysics Research Institute, Liverpool John Moores University, Twelve Quays House, \\ Egerton Wharf, Birkenhead CH41 1LD, UK \\ Received 2008 January 21; accepted 2008 May 27
}

\begin{abstract}
We present new results for the molecular gas, dust emission, and the ionized gas in J1023+1952, an H I-rich intergalactic star-forming tidal dwarf galaxy candidate. It is located at the projected intersection of two faint stellar tidal streams wrapped around the interacting pair of galaxies NGC 3227/6 (Arp 94). Using the IRAM $30 \mathrm{~m}$ telescope, emission from ${ }^{12} \mathrm{CO}(1-0)$ and ${ }^{12} \mathrm{CO}(2-1)$ was detected across the entire extent of the neutral hydrogen cloud associated with $\mathrm{J} 1023+1952$, a region of the size of $8.9 \times 5.9 \mathrm{kpc}$. The molecular gas is found to be abundant over the

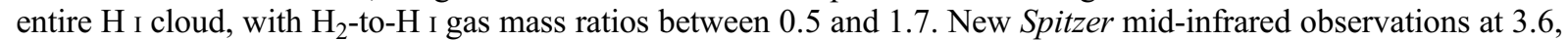
$4.5,5.8,8.0,15$, and $24 \mu \mathrm{m}$ show that young SF is restricted to the southern part of the cloud. Despite the relatively uniform $\mathrm{H}_{2}$ and $\mathrm{H}$ I column density across the cloud, young SF occurs only in those regions where the velocity dispersion in the $\mathrm{CO}$ and $\mathrm{H} \mathrm{I}$ is a factor of $\sim 2$ lower (FWHM of $30-70 \mathrm{~km} \mathrm{~s}^{-1}$ ) than elsewhere in the cloud (FWHM of $80-120 \mathrm{~km} \mathrm{~s}^{-1}$ ). Thus, the kinematics of the gas, in addition to its column density, seems to be a crucial factor in triggering SF. Optical/infrared spectral energy distributions (SEDs) and $\mathrm{H} \alpha$ photometry confirm that all the knots are young. Optical spectroscopy of the brightest SF region allowed us to determine the metallicity $[12+\log (\mathrm{O} / \mathrm{H})=$ $8.6 \pm 0.2]$ and the extinction $\left(A_{B}=2.4\right)$. This shows that $\mathrm{J} 1023+1952$ is made from metal-enriched gas which is inconsistent with the hypothesis that it represents a preexisting dwarf galaxy. Instead, it must be formed from recycled, metal-enriched gas, expelled from NGC 3227 or NGC 3226 in a previous phase of the interaction.
\end{abstract}

Subject headings: galaxies: active — galaxies: individual (Arp 94, J1023+1952, NGC 3227) galaxies: interactions — galaxies: ISM — molecular data

\section{INTRODUCTION}

Merger-driven galaxy evolution is important on a wide range of scales at different cosmic epochs and in different environments. The observed decline in the global star formation rate (SFR) at redshifts $z<2$ and the increased dominance of small galaxies in the local universe has been suggested to imply interaction-driven "downsizing" of galactic structure and star formation (Cowie et al. 1996; Madau et al. 1996; Brinchmann et al. 1998; Treu et al. 2005; Thomas et al. 2005) in contrast to hierarchical galaxy formation. The recently discovered free-floating intergalactic and extraplanar $\mathrm{H}$ II regions around nearby gas-rich galaxies (Gerhard et al. 2002; Ryan-Weber et al. 2004) and $\mathrm{H} \alpha$-emitting star-forming knots in galaxy clusters (Sakai et al. 2002) are the smallest intergalactic structures, and may represent an important evolutionary link or even a new mode of star formation (SF) in the low-density environment of the far outskirts of the galaxy halo. Their young

\footnotetext{
${ }^{1}$ Royal Society University Research Fellow.
}

star formation ages suggest they are tidal in origin, forming from gaseous tidal debris in intergalactic space.

The largest $\mathrm{H}$ II regions are typically found at the apparent tip of tidal tails, at more than $100 \mathrm{kpc}$ from the parent galaxies, in maxima of the $\mathrm{H}$ I column density where we also detect large quantities of molecular gas (Braine et al. 2000, 2001; Lisenfeld et al. 2002) and where enough tidal material is often available to build a dwarf galaxy (tidal dwarf galaxies, hereafter TDGs).

Indeed, tidal interactions between approximately equal-mass gas-rich galaxies have long been known to result in dramatic tidal damage, producing bridges and tails of debris extending out to several galaxy diameters (e.g., Toomre \& Toomre 1972; Haynes et al. 1984; Barnes \& Hernquist 1992; Mihos 2001; Mundell et al. 1995). Tidal tails often comprise primarily neutral hydrogen since $\mathrm{H} \mathrm{I}$ is usually the most spatially extended component of a spiral galaxy disk, and is therefore most easily stripped during the earliest stages of a tidal encounter (e.g., Hibbard \& van Gorkom 1996). Molecular gas is generally observed to be more centrally concentrated than $\mathrm{H}_{\mathrm{I}}$ in disks of galaxies outside clusters 


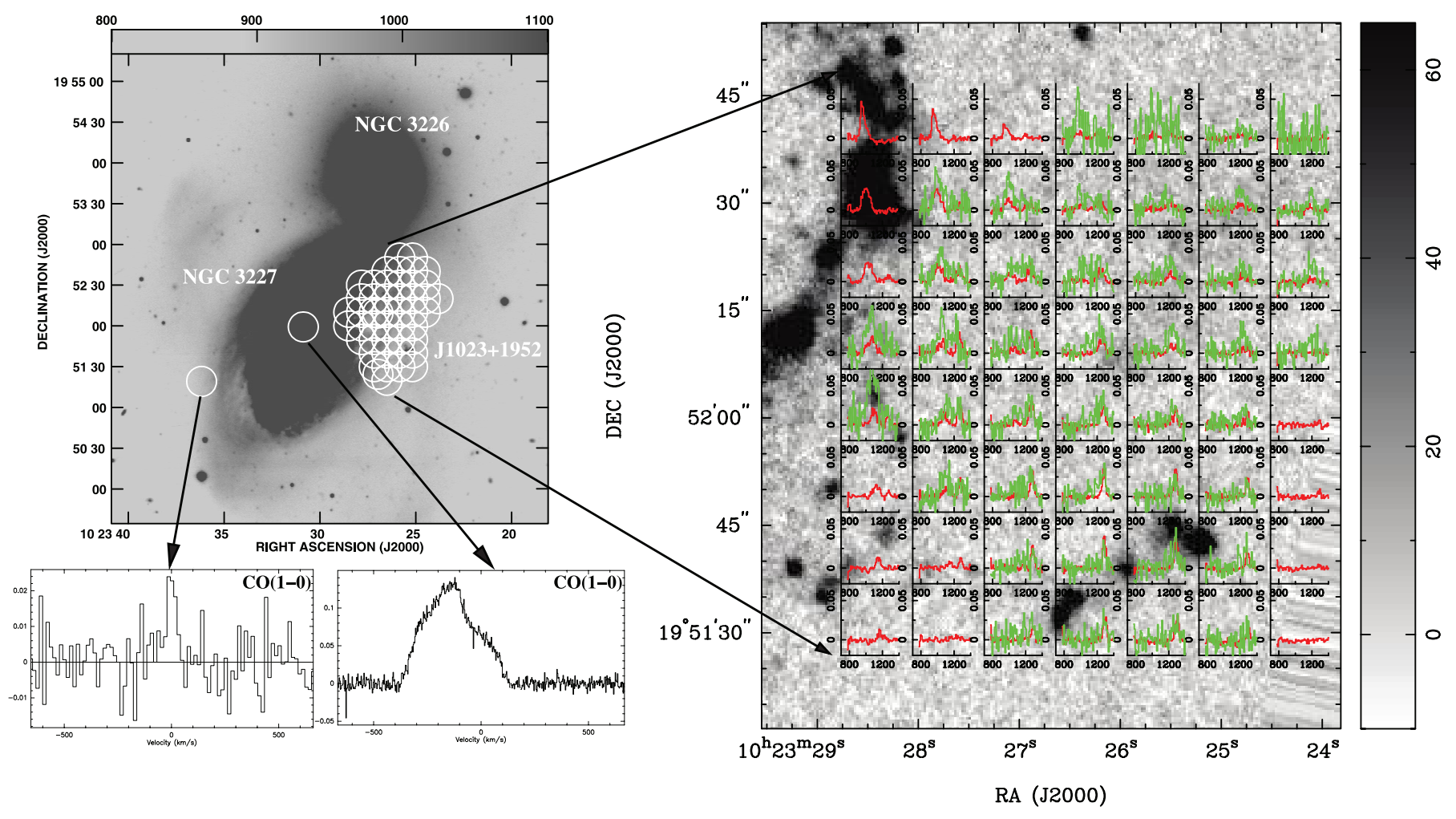

FIG. 1.-Left: Position of the IRAM pointings, overlaid on a blue image, together with the CO spectra of the positions observed within the disk of NGC 3227. Right: Spectra of $\mathrm{H}$ I emission (red lines) and ${ }^{12} \mathrm{CO}(1-0)$ emission (green lines) from $\mathrm{J} 1023+1952$ overlaid on an $\mathrm{H} \alpha$ image from Mundell et al. (2004).

(Nishiyama et al. 2001), so it is less likely to be stripped on first passage.

Here we present a study of an extragalactic region with active $\mathrm{SF}$ embedded in an extensive gas cloud named J1023+1952. The region is associated with the interacting Seyfert system Arp 94 (Mundell et al. 1995, 2004). Optically, the system is dominated by two disturbed Seyfert galaxies: NGC 3227, an SAB(s) pec barred spiral Seyfert galaxy, and its elliptical companion, NGC 3226 (E2 pec; Rubin \& Ford 1968; Mundell et al. 2004). Neutral hydrogen ( $\mathrm{H}_{\mathrm{I}}$ ) imaging of the system (Mundell et al. 1995) revealed two gaseous tidal tails extending $\sim 100 \mathrm{kpc}$ north and south of NGC 3227, well-ordered gas in the disk of NGC 3227 and a massive $\mathrm{H}$ I cloud $\left(M_{\mathrm{H}_{\mathrm{I}}}=3.8 \times 10^{8} M_{\odot}\right)$, that lies at the base of the northern tail and is close to, but physically and kinematically distinct from, the disk of NGC 3227. Mundell et al. (1995) suggested that this cloud (hereafter J1023+1952) might be a dwarf galaxy that is either preexisting and being accreted by Arp 94, or a newly created tidal dwarf galaxy (TDG) forming from the tidal debris.

The subsequent discovery of a region of very blue star-forming knots with luminosities between $10^{6}$ and $5 \times 10^{6} L_{\odot}$, embedded in a high $\mathrm{H}$ I column density ridge in the southern half of $\mathrm{J} 1023+1952$ (Mundell et al. 2004), confirmed its classification as a dwarf galaxy; the inferred SFR, UV luminosities, and $\mathrm{H} \alpha$ equivalent widths of the knots suggested a starburst age less than 10 Myr. Near-infrared imaging provided further evidence for the youth of $\mathrm{J} 1023+1952$; no additional embedded star formation or old stellar population were found (Mundell et al. 2004). These results suggest that the observed SF is currently being triggered in the interstellar medium (ISM) of J1023+1952 and does not merely represent young stars tidally stripped from the disk of NGC 3227 (Mundell et al. 2004).

The Arp 94 interacting system offers an interesting nearby laboratory in which to study the interplay between SF and the physics of the multiphase ISM in a tidally disrupted system with young, active intergalactic SF regions. Here we present a study of the optical, infrared, and millimeter properties of the Arp 94 system that aims to determine the nature and origin of J1023+ 1952 , its role in the observed interaction, and the underlying reasons for its observed star formation properties. Our data set comprises $(1){ }^{12} \mathrm{CO}(1-0)$ and ${ }^{12} \mathrm{CO}(2-1)$ mapping of the full extent of J1023+1923, the nucleus of NGC 3227, and the outer edges of the galactic disk of NGC 3227; (2) multiband infrared imaging with the Spitzer Space Telescope of J1023+1952 with particular emphasis on investigating the known SF regions and searching for further embedded SF; and (3) optical spectroscopy of the brightest $\mathrm{H}$ II region to determine the gas-phase metallicity.

The paper is structured as follows: the observations and data reduction are described in $\S 2$, molecular and atomic gas and star formation properties are derived in $\S 3$, and the implications for the triggering of SF and the origin of J1023+1952 as well as its role in the system are discussed in $\S 4$. We conclude in $\S 5$. Assuming a distance of $20.4 \mathrm{Mpc}$ to the Arp 94 system (Tully 1988), $1^{\prime \prime}$ corresponds to $\sim 100 \mathrm{pc}$.

\section{OBSERVATIONS AND ANALYSIS}

\subsection{CO Data}

We observed the ${ }^{12} \mathrm{CO}(1-0)$ and ${ }^{12} \mathrm{CO}(2-1)$ lines at 115 and $230 \mathrm{GHz}$ in 2004 June with the IRAM 30 m telescope on Pico Veleta. We fully mapped the H I extent of J1023+1952 and obtained two spectra toward NGC 3227 (see Fig. 1, left, for the positions of the individual pointings).

Dual polarization receivers were used at both frequencies with the $512 \times 1 \mathrm{MHz}$ filter banks on the $\mathrm{CO}(1-0)$ line and the $256 \times$ $4 \mathrm{MHz}$ filterbanks on the $\mathrm{CO}(2-1)$. Relatively high water vapor led to a high system temperature for the $230 \mathrm{GHz}$ data, and this degraded the signal-to-noise ratio $(\mathrm{S} / \mathrm{N})$ and baseline quality of the data. As a result, we could not further analyze the individual 
spectra; instead we averaged the emission in the $\mathrm{CO}(2-1)$ line over the whole cloud at this wavelength. The observations were done in wobbler switching mode with a wobbler throw of $200^{\prime \prime}$ in azimuthal direction. At the beginning of the observations the frequency tuning was checked by observing the central position of NGC 3227. Pointing was monitored on nearby quasars or Jupiter every 60-90 minutes. During the observation period, the weather conditions were generally good (with pointing better than $3^{\prime \prime}$ ), but we sometimes ( $\sim 4 \%$ of the total time) experienced periods of anomalous refractive index resulting in large $\left(>5^{\prime \prime}\right)$ pointing uncertainties. These data were excluded from our analysis. The average system temperature was $390 \mathrm{~K}$ at $115 \mathrm{GHz}$ on the $T_{A}^{*}$ scale. At $115 \mathrm{GHz}(230 \mathrm{GHz})$, the IRAM forward efficiency, $F_{\text {eff }}$, was $0.95(0.91)$, the beam efficiency, $B_{\text {eff }}$, was $0.75(0.54)$, and the half-power beam size $22^{\prime \prime}\left(11^{\prime \prime}\right)$. All CO spectra and luminosities are presented on the main beam temperature scale $\left(T_{\mathrm{mb}}\right)$ which is defined as $T_{\mathrm{mb}}=\left(F_{\text {eff }} / B_{\text {eff }}\right) T_{A}^{*}$. For the data reduction, we selected the observations taken during satisfactory weather condition (no anomalous refraction, low opacity), summed the spectra over the individual positions, and subtracted a constant continuum level.

Although the edges of the galactic disks of NGC 3227 and J1023+ 1952 spatially overlap in projection (Mundell et al. 1995, 2004), it is possible to disentangle their different kinematic components in the current ${ }^{12} \mathrm{CO}(1-0)$ data set. On average, the emission from NGC 3227 is blueshifted by approximately $200 \mathrm{~km} \mathrm{~s}^{-1}$ with respect to that of $\mathrm{J} 1023+1952$, with line widths not in excess of $100 \mathrm{~km} \mathrm{~s}^{-1}$, making it possible to separate them by fitting a double Gaussian line profile. The spectra were Hanning smoothed before the Gaussians were fitted. Initial fitting parameters were estimated by searching for maximum values and their associated positions in the spectra in two $150 \mathrm{~km} \mathrm{~s}^{-1}$ wide regions each centered on -200 and $0.0 \mathrm{~km} \mathrm{~s}^{-1}$ with respect to the systemic velocity of $\mathrm{J} 1023+1952$ of $1260 \mathrm{~km} \mathrm{~s}^{-1}$. Based on examination of the spectra by eye, the initial FWHMs of the lines were set to 180 and $120 \mathrm{~km} \mathrm{~s}^{-1}$ for NGC 3227 and J1023+1952, respectively.

As the two objects do not spatially overlap over the entire region mapped, three types of fit were undertaken, a simultaneous double fit, and two separate fits, each centered on the systemic velocity of one object. The $\chi^{2}$ values for each of the fits at each spatial pixel were calculated and were filtered according to the following criteria: (1) a line must be in emission (i.e., negative components are rejected as unphysical), (2) the line centers must lie in a window from -300 to $300 \mathrm{~km} \mathrm{~s}^{-1}$, (3) the FWHM must be greater than $18 \mathrm{~km} \mathrm{~s}^{-1}$ but less than $490 \mathrm{~km} \mathrm{~s}^{-1}$, and (4) the fit must be statistically significant at the $5 \sigma$ level or above. The significance limit was calculated from the noise measured at emission-free regions of the spectra and divided by the square root of the number of channels across the FWHM of the line. The median noise in the data was found to be $9.6 \mathrm{mK}$ per $2.6 \mathrm{~km} \mathrm{~s}^{-1}$ channel with mean values approaching zero, indicating good baselining. The fit with the lowest $\chi^{2}$ value meeting these criteria was then selected. The resulting fits for the individual components are presented in Figure 2, showing that satisfactory fits to the data could be achieved. The resulting difference between fit and data (lower part of each panel) was within the noise and showed a constant level over the whole velocity range. Further support for the correct decomposition of the $\mathrm{CO}$ lines comes from the good agreement between $\mathrm{CO}$ and $\mathrm{H}$ I line velocities and widths which will be discussed in $\S 3$.

\subsection{Optical Spectroscopy}

The optical spectra were obtained on 2007 May 9 with the instrument CAFOS at the $2.2 \mathrm{~m}$ telescope at Calar Alto. The slit had a fixed width of $1.2^{\prime \prime}$ and was placed with a position angle of $82^{\circ}$, crossing $\mathrm{H}$ II regions 1 and $1 \mathrm{a}$ (the labeling of the regions will be defined in $\S 3.3 .1$ ) and the nuclear region of NGC 3227. The spectra at knot 1a were too weak to derive the extinction or metallicity and are not taken into account. In order to cover the entire wavelength range, two spectra with different grisms, B100 (covering the range 3200-5800 $\AA$, with a dispersion of $2 \AA$ pixel $^{-1}$ ) and G100 (covering the range 4900-7800 $\AA$, with a dispersion of $2.12 \AA$ pixel $^{-1}$ ) were taken. The total integration time in each grism was 3600 and $3400 \mathrm{~s}$, respectively. The data reduction was carried out in a standard way using the IRAF software. The spectra were flux-calibrated using spectrophotometric standard stars, under nonphotometric, but transparent conditions suitable for relative line-flux measurements. An example of the final "red" spectrum is shown in Figure 3.

\subsection{Spitzer Data}

The Spitzer observations presented in this paper encompass observations covering both NGC 3226 and NGC 3227 which will be presented in P. N. Appleton et al. (in preparation). They were made early in the Spitzer mission with all three instruments, IRAC (2003 November 26), MIPS (2003 November 24), and IRS peak-up (2003 November 29). IRAC imaging in all four bands (3.6, 4.5, 5.8 , and $8 \mu \mathrm{m}$ ) was performed using a seven-point dither pattern to encompass both NGC 3227 and NGC 3226. Similarly, using a small-map photometry mode, images were made with MIPS at 24,70 , and $160 \mu \mathrm{m}$; however, only the images at $24 \mu \mathrm{m}$ were useful for $\mathrm{J} 1023+1952$ and are included in this study. Imaging was also performed using the IRS blue and red peak-up apertures (IRSPUI). IRAC and MIPS maps were made using the S14 (IRAC) and S15 (MIPS) data pipelines at the Spitzer Science Center. The MIPS results were checked by performing off-line mosaicing with different methods of background subtraction, but the results were similar to those obtained in the pipeline. An exception was the IRS peak-up imaging in which the final image was created by running basic calibrated data frames (BCDs) created in the S14 IRS pipeline offline using the SSC MOPEX software. This produced good results for the background in the region of $\mathrm{J} 1023+1952$.

At $24 \mu \mathrm{m}$ the central AGN of NGC 3227 was so strong that its point-spread function (PSF) extended out to the region of $\mathrm{J} 1023+$ 1952 , visible in symmetrical radial fingers. We corrected for this by subtracting a point source convolved with the PSF of MIPS at the position of the central AGN. The removal of the PSF of the AGN at $24 \mu \mathrm{m}$ resulted in a roughly constant decrease in flux from the direction of $\mathrm{J} 1023+1952$ of approximately $30 \%$, and this is taken into account in the uncertainties quoted. In Figure 4 the Arp 94 image at $24 \mu \mathrm{m}$ before and after the AGN subtraction is shown. At $16 \mu \mathrm{m}$ the PSF of the bright AGN was also visible but did not extend into the area of the $\mathrm{H}$ I cloud so that the original image could be used.

The $8 \mu \mathrm{m}$ emission contains emission from stellar continuum as well as dust emission, mainly from PAH emission. We estimate the contribution from dust by subtracting the stellar emission, applying the formulae given in Pérez-González et al. (2006). They followed the procedure described in Pahre et al. (2004), who used a Vega star template to extrapolate the stellar light from 3.5/4.5 to $8 \mu \mathrm{m}$. We found that in all individual knots the $8 \mu \mathrm{m}$ emission was almost entirely $(>90 \%)$ due to dust. Therefore, we neglect in the following a possible small contamination from stellar light and assume that the entire $8 \mu \mathrm{m}$ emission is from dust.

\section{RESULTS}

\subsection{Molecular and Atomic Gas}

\subsubsection{Gas Distribution}

Figure 1 (right) shows ${ }^{12} \mathrm{CO}(1-0)$ spectra (green lines) taken across the Arp 94 system, superimposed on an $\mathrm{H} \alpha$ image in which 

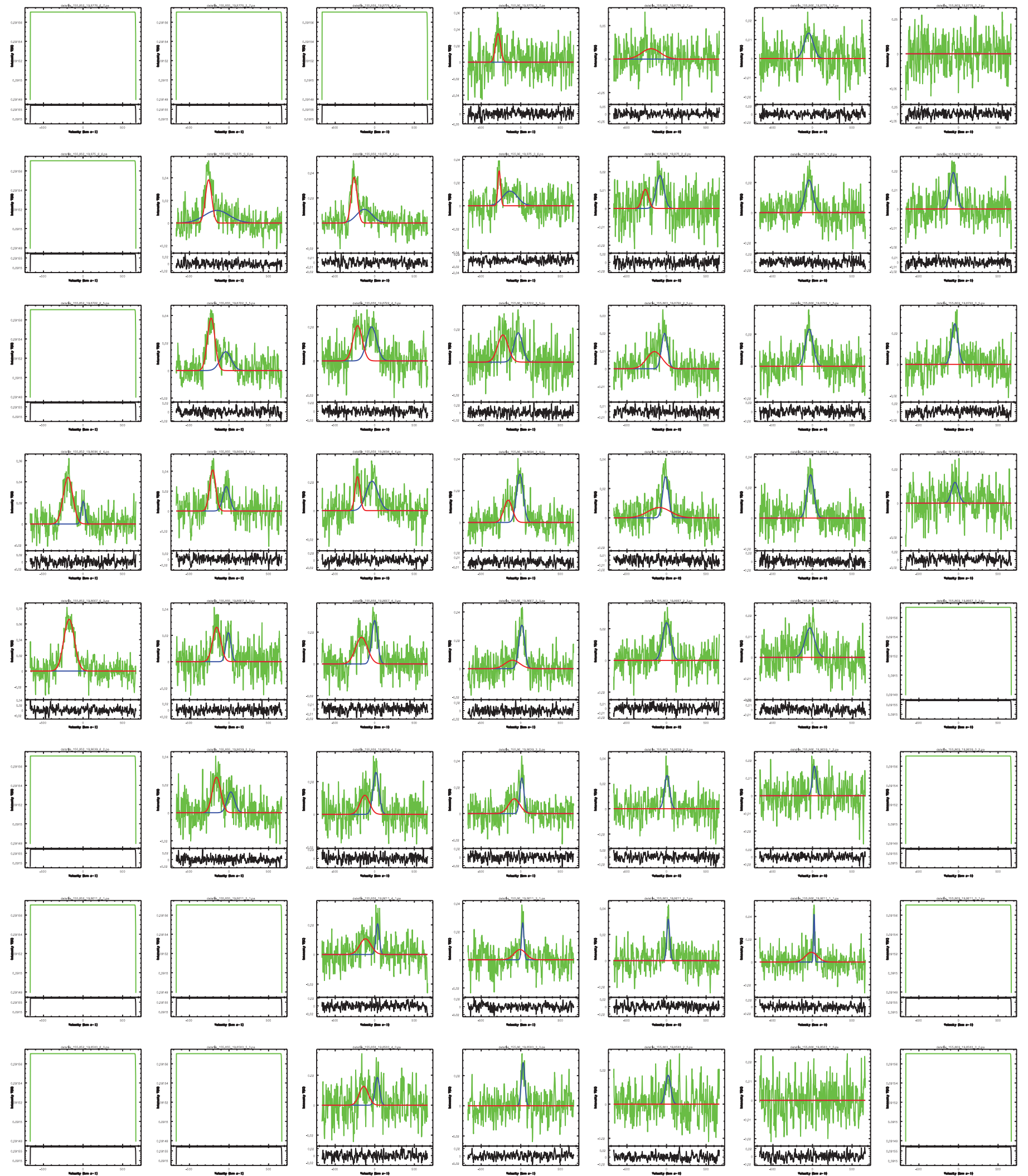

FIg. 2.-Observed spectra ( green lines) together with their Gaussian fits. The red line shows the fit to the component from the disk of NGC 3227 and the blue line to the component of J1023+1952. The velocity scale is relative to a systemic velocity of $1260 \mathrm{~km} \mathrm{~s}^{-1}$. The individual panels correspond to the observed positions shown in Fig. 1. The lower plot in each panel shows the difference between the two-component fit and the data. Note the narrower line widths coincide with the star-forming knots in J1023+1952 (bottom two rows of spectra, see Fig. 1). 


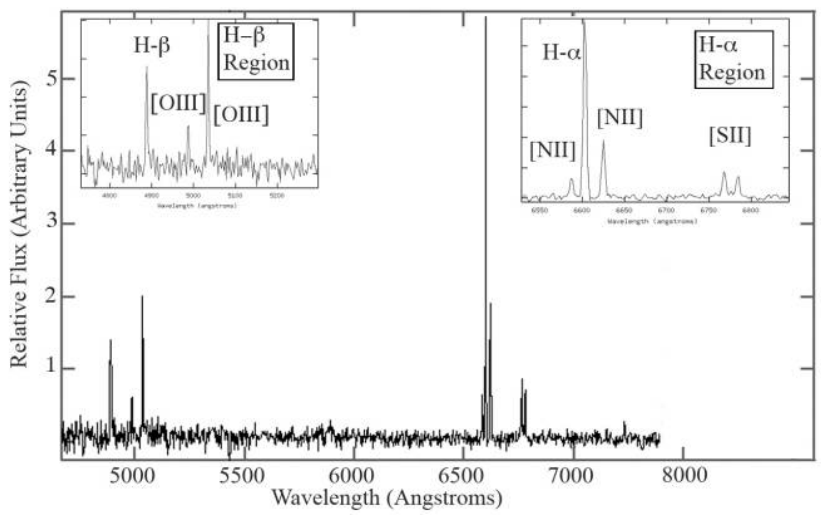

FIG. 3.-Optical spectrum at the position of the knot 1. The insets show blown-up versions of the relevant regions around the $\mathrm{H} \alpha$ and $\mathrm{H} \beta$ line.

the disk of NGC 3227 and the star-forming knots in J1023+1952 can be seen. Emission from ${ }^{12} \mathrm{CO}(1-0)$ is detected (1) across the entire $\sim 9 \times 6 \mathrm{kpc} \mathrm{H}$ I extent of $\mathrm{J} 1023+1952$, not only in the starforming regions, (2) from the well-studied gas-rich Seyfert nucleus of NGC 3227 as expected (Schinnerer et al. 2000), and (3) at the outer edges of the disk of NGC 3227 at a radius of $\sim 9 \mathrm{kpc}$ to the SE and NW of the nucleus. Also shown for comparison in Figure 1 (right) are the corresponding $\mathrm{H}$ i spectra (red line) at these locations.

Figure 5 shows the velocity integrated intensity of the $\mathrm{CO}$ emission (which is proportional to the molecular gas column density) of $\mathrm{J} 1023+1952$ alone (after separating out the emission of NGC 3227 based on the Gaussian fit, as explained in $\S 2.1$ ), overlaid with contours of the $\mathrm{H}$ I surface density at a spatial resolution of $6.3^{\prime \prime} \times 6.3^{\prime \prime}$. It is interesting that the molecular gas surface density in the northern part is even higher than in the southern part. The distributions of $\mathrm{H}$ i and $\mathrm{H}_{2}$ show differences: the $\mathrm{H}$ I peaks in a ridge-like structure toward the east which is not followed by the $\mathrm{H}_{2}$, but which presents instead a decrease in surface density from north to south. In order to quantify these trends, we list in Table 1 the column densities of the molecular gas [assuming a standard Galactic conversion factor of $N\left(\mathrm{H}_{2}\right)$ / $\left.I_{\mathrm{CO}}=2 \times 10^{20} \mathrm{~cm}^{-2}\left(\mathrm{~K} \mathrm{~km} \mathrm{~s}^{-1}\right)^{-1}\right]$, atomic gas and total gas, as well as the ratio between the $\mathrm{H}_{2}$ and $\mathrm{H}$ i column densities (molecular gas fraction) for the three regions labeled in Figure 5. These regions correspond to (1) the southern SF region, (2) a region where both the atomic and molecular gas surface density are high, and (3) a region with low atomic, but high molecular surface density. To derive these values we used the $\mathrm{H}$ I $\mathrm{C}$-array data from Mundell et al. (1995) which had a spatial resolution of $20.47^{\prime \prime} \times$ $18.25^{\prime \prime}$, comparable to that of the CO data.

Surprisingly, the lowest molecular surface density and molecular fraction are found in region 1 , the SF region. This shows that a high molecular (or total) gas column density is not enough to predict whether SF takes place or not. The H i column density, on the other hand, has a peak in region 1.

\subsubsection{CO Line Ratio}

Emission from ${ }^{12} \mathrm{CO}(2-1)$ was also detected from $\mathrm{J} 1023+1952$ at most locations, although with a poorer $\mathrm{S} / \mathrm{N}$ than ${ }^{12} \mathrm{CO}(1-0)$, but sufficient to calculate a mean ratio ${ }^{12} \mathrm{CO}(2-1) /{ }^{12} \mathrm{CO}(1-0)$, averaged over the whole object. Since the $\mathrm{S} / \mathrm{N}$ of the ${ }^{12} \mathrm{CO}(2-1)$ data was too low to perform the separation between the emission of J1023+1952 and NGC 3227, we made the approximation that emission below $-120 \mathrm{~km} \mathrm{~s}^{-1}$ [for both ${ }^{12} \mathrm{CO}(1-0)$ and ${ }^{12} \mathrm{CO}(2-1)$ ] was from NGC 3227, and emission above this value from $\mathrm{J} 1023+$ 1952. In this way, we derived an average ratio ${ }^{12} \mathrm{CO}(2-1) /$ ${ }^{12} \mathrm{CO}(1-0)=0.54 \pm 0.10$, where the error takes into account both the error due to the rms noise of the spectra and a calibration uncertainty of $10 \%$. We do not take into account a possible error due to the undersampling of the ${ }^{12} \mathrm{CO}(2-1)$ emission with our $10^{\prime \prime}$ spacing. The line ratio is practically unchanged when including the emission of NGC 3227, which shows that there is no significant difference between the line ratios of J1023+1952 and the outer disk of NGC 3227. This ratio is lower than the mean value found in the central regions of spiral galaxies (e.g., $0.89 \pm$ 0.06, with a dispersion of 0.34; Braine \& Combes 1992). The low line ratio could indicate a relatively low gas density, leading to a subthermal excitation of the ${ }^{12} \mathrm{CO}(2-1)$ line. Due to the different beams of the ${ }^{12} \mathrm{CO}(2-1)$ and ${ }^{12} \mathrm{CO}(1-0)$ spectra and the undersampling of the ${ }^{12} \mathrm{CO}(2-1)$ emission, we were not able to study local variations of the line ratio.

\subsubsection{Gas Kinematics}

In Figure 6 we show the intensity-weighted radial velocities. These velocities show a smooth gradient over J1023+1952 with
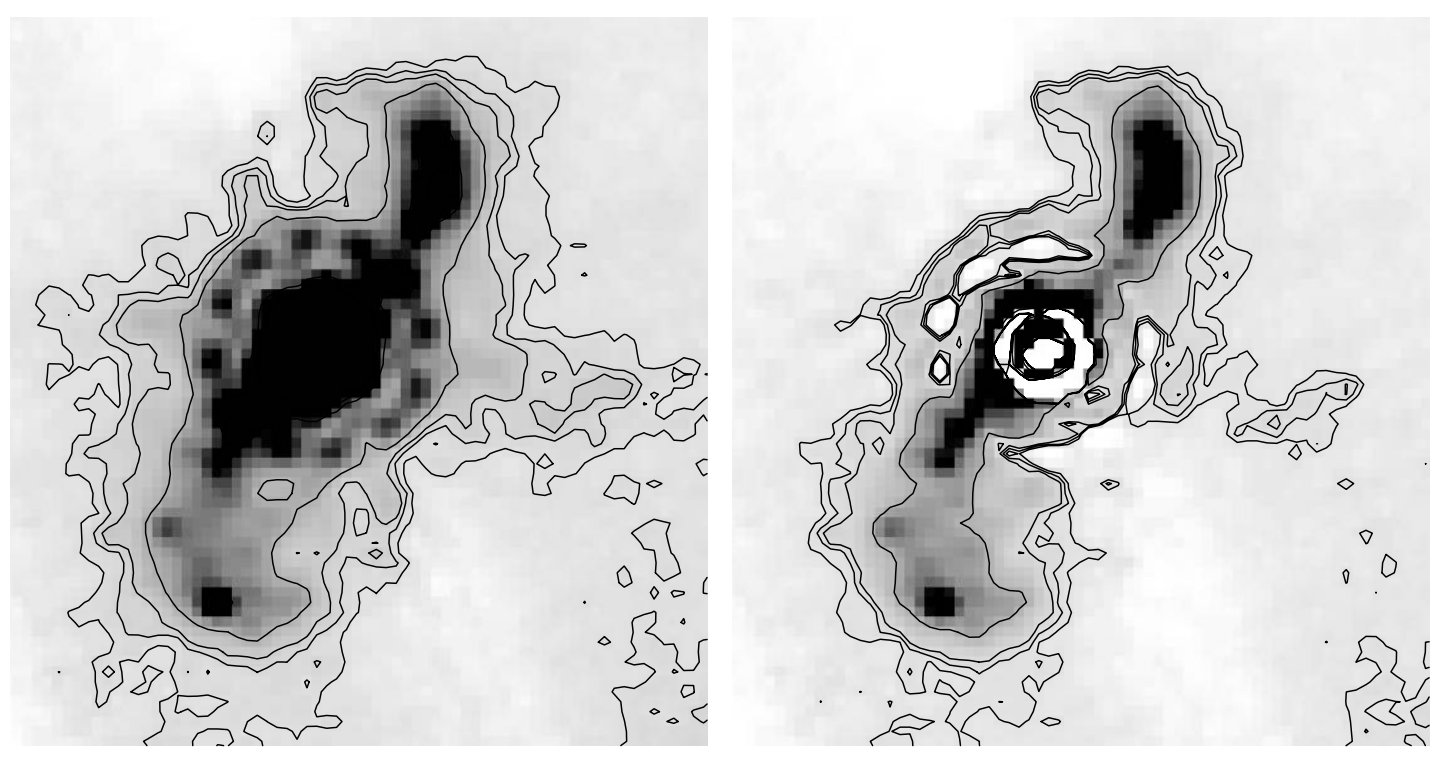

FIG. 4. - Left: $24 \mu \mathrm{m}$ image of NGC 3227 and J1023+1952. Right: Sidelobe emission from the strong nucleus of NGC 3227 has been subtracted and the emission at the $\mathrm{SF}$ region of J1023+1952 is now clearly visible. 


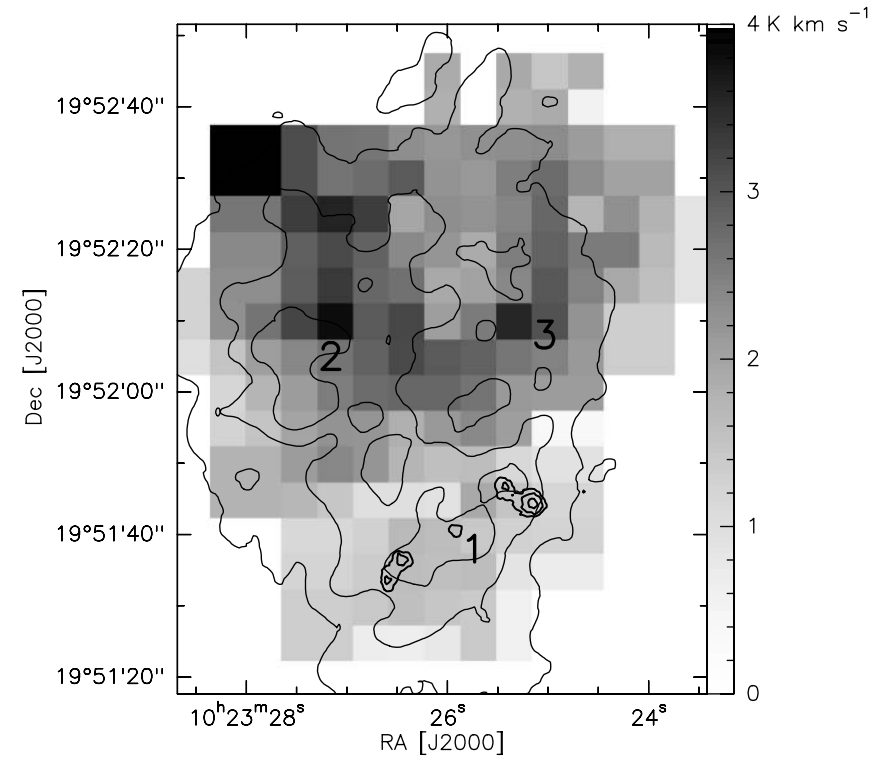

FIG. 5. - Velocity-integrated intensities (moment 0 ) of $\mathrm{CO}$ (gray areas); $1 \mathrm{~K} \mathrm{~km} \mathrm{~s}^{-1}$ correspond to a surface density of $2 \times 10^{20} \mathrm{~cm}^{-2}$. Overlaid (contour) is the moment 0 map of $\mathrm{H}_{\mathrm{I}}$ (Mundell et al. 2004), observed with the VLA in $B$-array with a spatial resolution of $6.3^{\prime \prime} \times 6.3^{\prime \prime}$. The contour levels are 10,40 , and $70 \mathrm{Jy}$ beam ${ }^{-1} \mathrm{~ms}^{-1}$, corresponding to $0.3,1.1$, and $2.0 \times 10^{21} \mathrm{~cm}^{-2}$. The numbers indicate positions discussed separately in the text. The thick contours show the $\mathrm{H} \alpha$ emission.

a maximum shift of about $80 \mathrm{~km} \mathrm{~s}^{-1}$ from the north to the south over a distance of about $70^{\prime \prime}(6.9 \mathrm{kpc})$. The gradient agrees very well with that seen in the $\mathrm{H}$ I line (see Fig. $8 b$ in Mundell et al. 1995). The agreement in the velocity fields derived for the CO and $\mathrm{H}$ i provides further confidence that the profile decomposition between the CO emission from J1023+1952 and NGC 3227 in the northeast of the cloud was successful.

Figure 7 shows the line velocity widths (FWHM) of the ${ }^{12} \mathrm{CO}(1-0)$ emission. The line widths are smallest in the southern SF region (region 1) with values for the FWZI around $100 \mathrm{~km} \mathrm{~s}^{-1}$ and FWHM of the Gaussian fit between 30 and $70 \mathrm{~km} \mathrm{~s}^{-1}$. Northwards of this area (region 3), the line widths become substantially larger, with FWZI around $200 \mathrm{~km} \mathrm{~s}^{-1}$ and FWHM of the Gaussian fit between 80 and $120 \mathrm{~km} \mathrm{~s}^{-1}$. Also the area around the H I peak (region 2) shows broad lines, with FWHM of 80$90 \mathrm{~km} \mathrm{~s}^{-1}$. The same trends hold for the $\mathrm{H}$ emission, since the $\mathrm{H} \mathrm{I}$ and CO spectra agree very well in velocity and width (see Fig. 1).

\subsubsection{Gas Masses}

Assuming a standard Galactic conversion factor of $N\left(\mathrm{H}_{2}\right)$ / $I_{\mathrm{CO}}=2 \times 10^{20} \mathrm{~cm}^{-2}\left(\mathrm{~K} \mathrm{~km} \mathrm{~s}^{-1}\right)^{-1}$, the total molecular gas mass can be calculated via $M_{\mathrm{H}_{2}}\left[M_{\odot}\right]=75 I_{\mathrm{CO}} D^{2} \Omega$ (Lisenfeld et al. 2002), where $I_{\mathrm{CO}}$ is the velocity-integrated $\mathrm{CO}$ line intensity in $\mathrm{K} \mathrm{km} \mathrm{s}^{-1}$, $D$ is distance in Mpc, and $\Omega$ is the area in $\operatorname{arcsec}^{2}$. The integrated intensity for $\mathrm{J} 1023+1952$, over the extent of the $\mathrm{CO}$ emission of $\sim 90^{\prime \prime} \times 60^{\prime \prime}$ (corresponding to $8.9 \times 5.9 \mathrm{kpc}$ ) is $1.3 \mathrm{~K} \mathrm{~km} \mathrm{~s}^{-1}$, resulting in a total molecular gas mass $M_{\mathrm{H}_{2}} \sim 2.2 \times 10^{8} M_{\odot}$ (including a helium fraction of $\left.1.38, M_{\mathrm{H}_{2}+\mathrm{He}} \sim 3.0 \times 10^{8} M_{\odot}\right)$. The total mass of the atomic gas is $M_{\mathrm{H}_{\mathrm{I}}}=3.8 \times 10^{8} M_{\odot}$ (Mundell et al. 2004), giving a rather high molecular-to-atomic gas mass fraction of $M_{\mathrm{H}_{2}} / M_{\mathrm{H}_{\mathrm{I}}}=0.6$.

Including only narrow-line emission from the vicinity of the star-forming knots results in $M_{\mathrm{H}_{2}+\mathrm{He}} \sim 1.05 \times 10^{8} M_{\odot}$. The SFR in this region, derived from the $\mathrm{H} \alpha$ emission (extinction corrected with $A_{\mathrm{H} \alpha}=1.4 \mathrm{mag}$; see $\left.\S 3.2\right)$ is $3.6 \times 10^{-2} M_{\odot} \mathrm{yr}^{-1}$, which yields a gas consumption time of $3 \times 10^{9} \mathrm{yr}$. Taking also atomic gas into account, the gas consumption time increases by a factor of 2.3. This long timescale indicates that plenty of gas is available to drive SF for an extended period of time.

\subsection{Extinction and Metallicity}

Line fluxes were measured in the optical spectrum shown in Figure 3 using the IRAF splot procedure. We used the $\mathrm{H} \alpha / \mathrm{H} \beta$ ratio to derive the extinction assuming case $\mathrm{B}$ recombination of Osterbrock (1989), which predicts an intrinsic $\mathrm{H} \alpha / \mathrm{H} \beta$ of 2.86 for an electron temperature of 10,000 K. We derived an extinction of $A_{\mathrm{H} \alpha}=1.4 \mathrm{mag}$. Based on this value and the extinction curve given in Draine (2003; his Table 4), we dereddened the lines. The resulting, dereddened line fluxes, relative to $\mathrm{H} \beta$, are listed in Table 2.

We derived the oxygen abundance based on the standard empirical $R 23$ method (Pagel et al. 1979). From log ([O III $]_{\lambda 5007} /$ $\left.\left[\mathrm{N}_{\text {III }}\right]_{\lambda 6584}\right)=0.2$ we determined (e.g., from Edmunds \& Pagel 1984) that we are in the upper branch of the $R 23-12+\log (\mathrm{O} / \mathrm{H})$ relation. The value of $R 23=\left([\mathrm{O} \text { III }]_{\lambda 5007}+[\mathrm{O} \text { III }]_{\lambda 4959}+\left[\mathrm{O}_{\text {II }}\right]_{\lambda 3737}\right) /$ $\mathrm{H} \beta)=6.2$ yields then $12+\log (\mathrm{O} / \mathrm{H})=8.6 \pm 0.2$. We also applied the empirical method of Denicoló et al. (2002) which is based on the nitrogen-to- $\mathrm{H} \alpha$ ratio and derived from $\log \left(\left[\mathrm{N} \mathrm{III}_{\lambda 6584} / \mathrm{H} \alpha\right)=\right.$ -0.5 practically the same oxygen abundance, $12+\log (\mathrm{O} / \mathrm{H})=$ $8.7 \pm 0.2$. This metallicity is higher than what is typically found in dwarf galaxies, and more similar to values measured in disks of spiral galaxies [e.g., the solar metallicity is $12+\log (\mathrm{O} / \mathrm{H})=$ 8.66; Asplund et al. 2005].

\subsection{Star Formation in J1023+1952}

\subsubsection{Spitzer Mid-IR Emission}

At all observed wavelengths, the only obvious emission within $\mathrm{J} 1023+1952$ is from the SF knots in the south. Figure $8 a$ shows the optical $B$-band image with the linear arrangement of bright blue knots labeled 1, 2, 3, 4, and 5, according to Mundell et al. (2004); we also label additional knots, 1a and 2a, which were visible in the images of Mundell et al. (2004), but not specifically identified by them. In Figs. $8 b-8 f$ we show comparisons between Spitzer emission at $8 \mu \mathrm{m}$ (in contours), the blue, $\mathrm{H} \alpha$ and Spitzer

TABLE 1

Surface Densities in Various Regions of J1023+1952

\begin{tabular}{|c|c|c|c|c|}
\hline Region $^{\mathrm{a}}$ & $\begin{array}{c}N\left(\mathrm{H}_{2}\right) \\
\left(10^{20} \mathrm{~cm}^{-2}\right)\end{array}$ & $\begin{array}{c}N(\mathrm{H} \mathrm{I})^{\mathrm{b}} \\
\left(10^{20} \mathrm{~cm}^{-2}\right)\end{array}$ & $2 N\left(\mathrm{H}_{2}\right) / N\left(\mathrm{H} \mathrm{I}_{\mathrm{I}}\right)$ & $\begin{array}{c}2 N\left(\mathrm{H}_{2}\right)+N(\mathrm{H} \mathrm{I} \mathrm{I}) \\
\left(10^{20} \mathrm{~cm}^{-2}\right)\end{array}$ \\
\hline 1 & 3 & 12 & 0.5 & 18 \\
\hline $2 \ldots \ldots \ldots+\ldots$ & 6 & 15 & 0.8 & 27 \\
\hline З & 5 & 6 & 1.7 & 16 \\
\hline
\end{tabular}

a The positions of the region are indicated in Fig. 5.

b The H I surface density was obtained from the H I C-array data from Mundell et al. (1995) which have a similar spatial resolution $\left(20.47^{\prime \prime} \times 18.25^{\prime \prime}\right)$ as the $\mathrm{CO}$ data. 


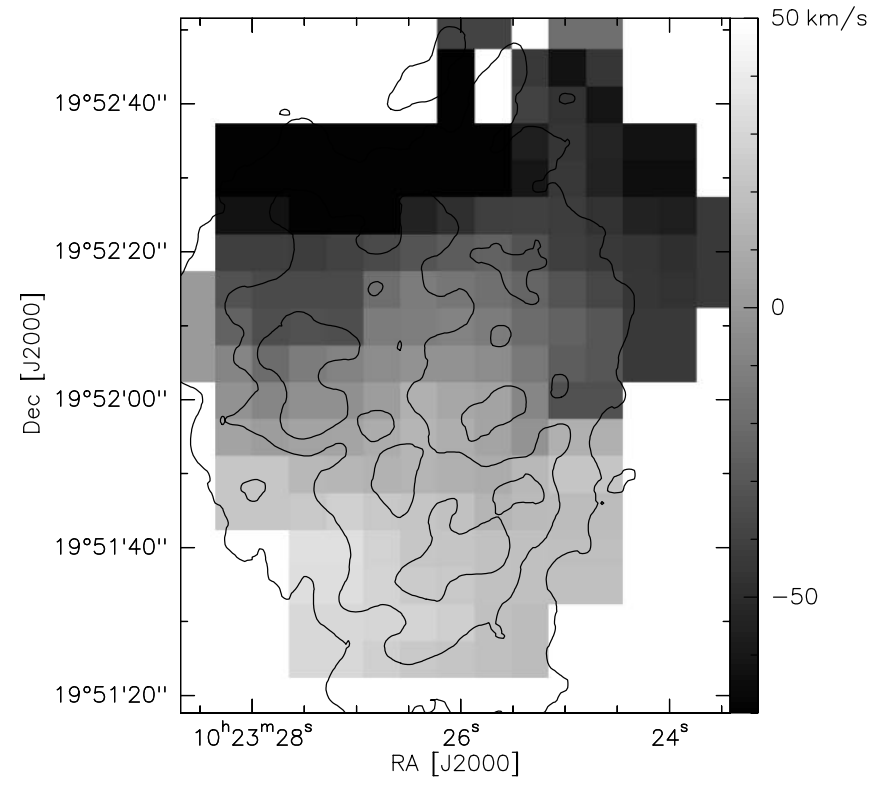

FIG. 6.- Intensity-weighted radial velocity (moment 1 ) of CO (gray areas) over-

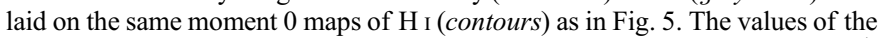
intensity-weighted radial velocities are relative to a systemic velocity of $1260 \mathrm{~km} \mathrm{~s}^{-1}$.

emissions at 3.6, 15, and $24 \mu \mathrm{m}$. In general, a good structural agreement between the emissions at all wavelengths can be seen (exceptions will be discussed below), but with a different relative strengths. In Table 3 we list the integrated fluxes of the individual knots with detected emission.

For most knots, there is a very good agreement between the emission at $8 \mu \mathrm{m}, \mathrm{H} \alpha$, and the blue. A striking aspect is the absence of $8 \mu \mathrm{m}$ ( $\mathrm{PAH}+$ dust continuum) emission from the brightest optical knot 2 and its strong decrease or absence at knot 5 . The dust emission seems to be also missing at longer wavelengths, albeit traced with a poorer spatial resolution. The lack of $8 \mu \mathrm{m}$ emission is accompanied by a lack of $\mathrm{H} \alpha$ in knot 2, but not in knot 5, which shows strong $\mathrm{H} \alpha$ emission.

The $3.6 \mu \mathrm{m}$ emission, usually associated with the photospheres of older stars, is visible from all knots, with the strongest

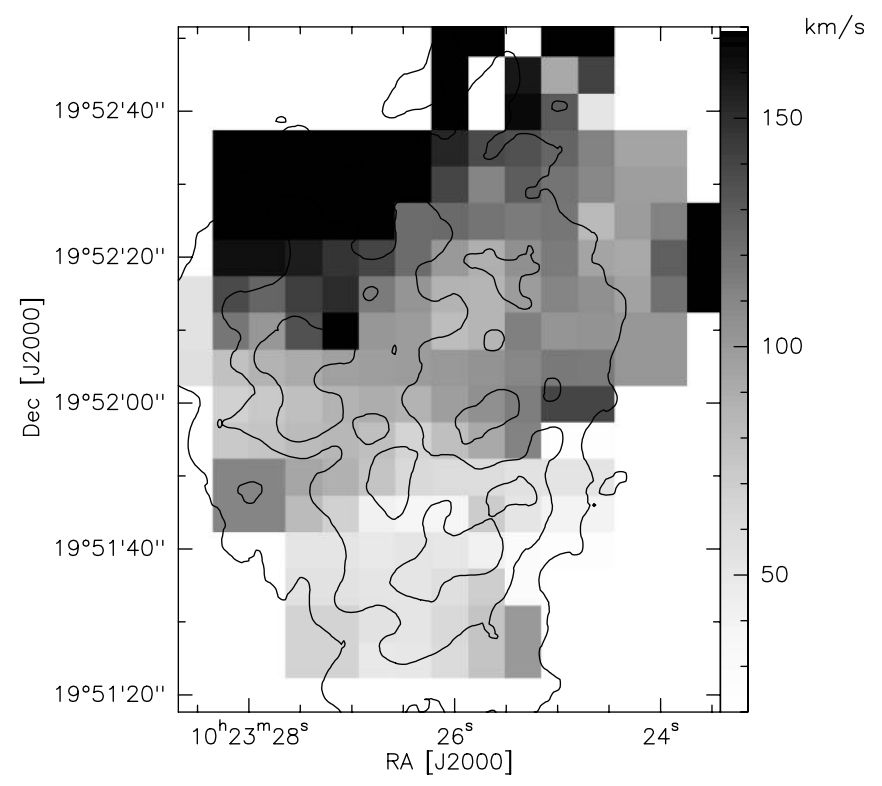

FIG. 7.-FWHM of the CO lines (gray areas) overlaid on the same moment 0 maps of $\mathrm{H}$ I (contours) as in Fig. 5.
TABLE 2

Extinction-Corrected Line Intensities of Knot 1 with Respect to $\mathrm{H} \beta=100$

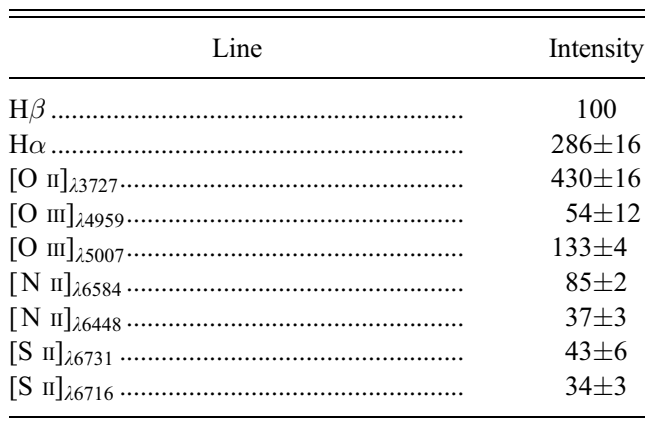

emission coming from knots 4,5 , and $2 \mathrm{a}$. The detected emission is associated only with the knots and no additional smoothly distributed emission is detected, thus ruling out the presence of an extended underlying old stellar population. This confirms the previous nondetection of an extended component at near-IR wavelengths by Mundell et al. (2004). The emissions at 15 and $24 \mu \mathrm{m}$ are also clearly detected, but with a poorer resolution and $\mathrm{S} / \mathrm{N}$.

\subsubsection{The Optical/MIR SEDs of the Star-forming Knots}

We carried out aperture photometry of the SF knots on images at optical wavelengths ( $B, V$, and $I$ bands), $\mathrm{H} \alpha$ (data from Mundell et al. 2004), and the Spitzer IRAC bands and $15 \mu \mathrm{m}$ emission. Due to the poorer spatial resolution, we could not derive the $24 \mu \mathrm{m}$ fluxes for the individual knots. For the aperture photometry, we smoothed the optical images to the resolution of the $8 \mu \mathrm{m}$ image $\left(2^{\prime \prime}\right)$ and carried out photometry on this set of images. We used a circular aperture with a radius of $3.6^{\prime \prime}$ centered on the individual knots indicated in Figure 8. Because of their small distance, knots 4 and 5 could just barely be distinguished individually. The integrated fluxes derived in the aperture photometry are given in Table 3.

We show in Figure 9 the optical-IR SEDs for the individual knots. For knot 1 we measured the extinction $\left(A_{\mathrm{H} \alpha}=1.4 \mathrm{mag}\right.$, corresponding to $A_{B}=2.4 \mathrm{mag}$ ), so that we can show both the extinction corrected and uncorrected SED for this knot.

The knots show clear differences in their SEDs, most likely due to differences in their SF history and presence of dust. In the following, we discuss them individually. We compared the data to simulations with Starburst99 (Leitherer et al. 1999), using both continuous and instantaneous star formation, and assuming solar metallicity and a Salpeter initial mass function, in order to set constraints on the age and extinction of the star-forming knots.

1. Knot 1 gives a consistent picture of a young star-forming region: the extinction-corrected optical SED shows a very blue color and $\mathrm{H} \alpha$ and dust emission is present. The simulations with Starburst99 yielded ages between 1 and 2 Myr (both for a continous and instantaneous burst) for this knot.

2. The uncorrected SEDs of knots $1 \mathrm{a}$ and 3 are very similar to that of knot 1 and they also present $\mathrm{H} \alpha$ and dust emission. Thus, if the dust extinction for these knots is similar to that in knot 1 , which is plausible given the small overall variation of the $\mathrm{H} \mathrm{I}$ column density across the SF region (Mundell et al. 2004), they are also representing regions of very young SF with ages between 1 and 2 Myr. The shape of the SED of these knots allows us to constrain the minimum extinction: The peak in the $V$ band of the unextincted SED cannot be produced by any synthetic SEDs of Starburst99. We need a minimum extinction of $A_{B}=1.9$ mag in 

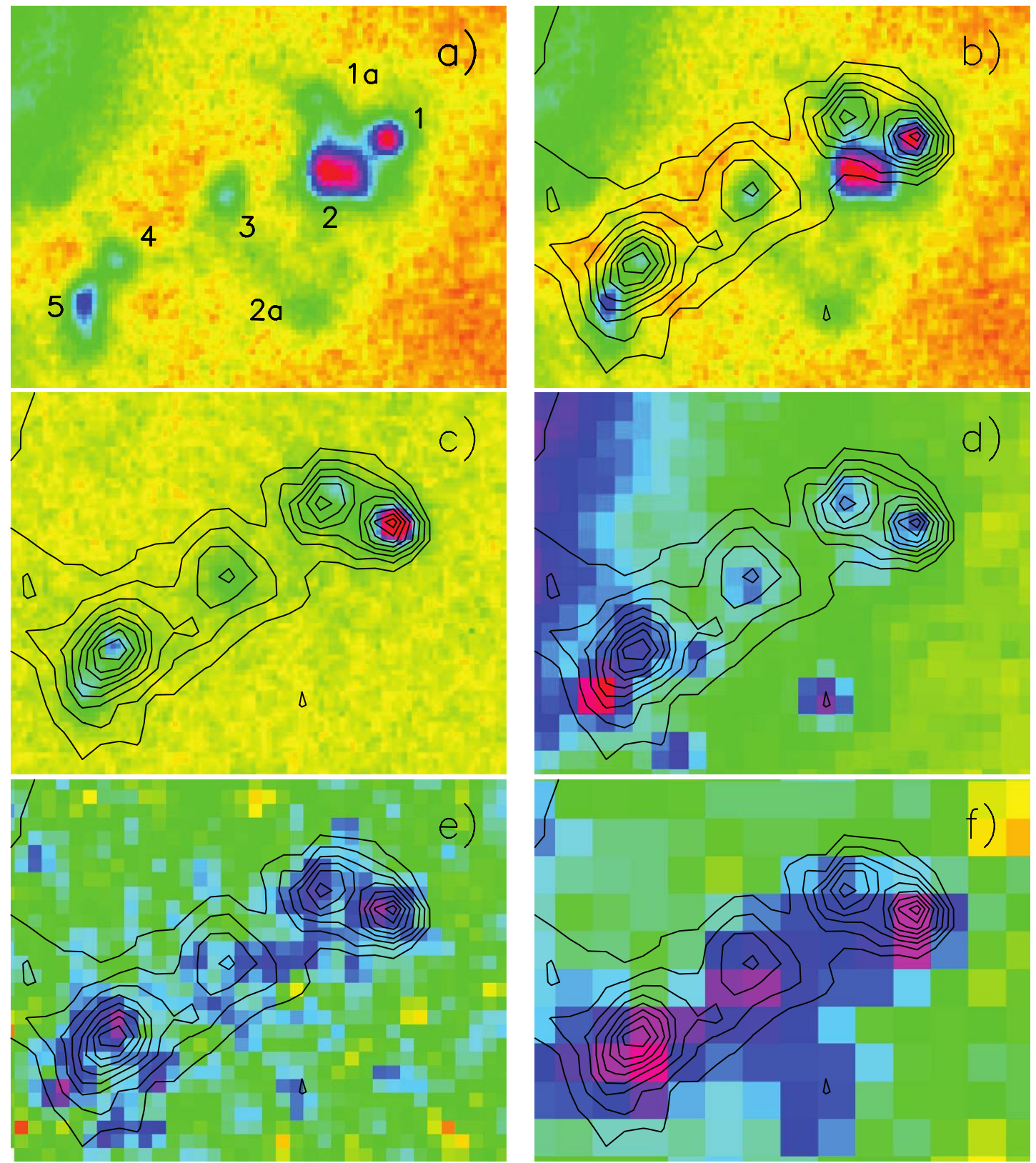

FIG. 8. - Spitzer observations of J1023+1952: (a) the $B$-band image indicating the numbers of the respective knots. The other panels show the $8 \mu \mathrm{m}$ contours superimposed on $(b)$ the $B$-band image, $(c)$ the $\mathrm{H} \alpha$ image, $(d)$ the $3.5 \mu \mathrm{m}$ image, $(e)$ the $15 \mu \mathrm{m}$ image, and $(f)$ the $24 \mu \mathrm{m}$ image.

TABLE 3

Aperture Integrated Optical and IR Flux Densities of the Star-Forming Knots

\begin{tabular}{|c|c|c|c|c|c|c|c|c|c|c|c|}
\hline Knot & $\begin{array}{l}r_{\text {aperture }} \\
(\operatorname{arcsec})\end{array}$ & $\begin{array}{c}S_{B} \\
(\mu \mathrm{Jy})\end{array}$ & $\begin{array}{c}S_{R} \\
(\mu \mathrm{Jy})\end{array}$ & $\begin{array}{c}S_{I} \\
(\mu \mathrm{Jy})\end{array}$ & $\begin{array}{l}S_{3.6} \\
(\mu \mathrm{Jy})\end{array}$ & $\begin{array}{c}S_{4.5} \\
(\mu \mathrm{Jy})\end{array}$ & $\begin{array}{l}S_{5.8} \\
(\mu \mathrm{Jy})\end{array}$ & $\begin{array}{l}S_{8.0} \\
(\mu \mathrm{Jy})\end{array}$ & $\begin{array}{l}S_{15} \\
(\mu \mathrm{Jy})\end{array}$ & $\begin{array}{c}S_{24} \\
(\mu \mathrm{Jy})\end{array}$ & $\begin{array}{c}S_{\mathrm{H} \alpha} \\
\left(10^{-15} \operatorname{ergs~s}^{-1} \mathrm{~cm}^{-2}\right)\end{array}$ \\
\hline 1 & 3.6 & 39.3 & 44.9 & 25.8 & 49.3 & 40.8 & 183 & 505 & 376 & $\ldots$ & 9.6 \\
\hline 2 & 3.6 & 60.9 & 59.2 & $\ldots$ & 54.4 & 41.6 & $\ldots$ & $\ldots$ & $\ldots$ & $\ldots$ & $\ldots$ \\
\hline 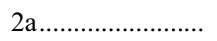 & 3.6 & 12.6 & 24.2 & 27.2 & 48.5 & 34.7 & 80.9 & 118 & $\ldots$ & $\ldots$ & $\ldots$ \\
\hline 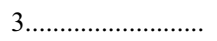 & 3.6 & 13.3 & 26.0 & 20.3 & 18.1 & 25.8 & 156 & $404 / 590^{a}$ & 363 & $855^{\mathrm{a}}$ & $2.9 / 3.8^{\mathrm{a}}$ \\
\hline $1+1 \mathrm{a} \ldots \ldots \ldots \ldots$ & 7.2 & $\ldots$ & $\ldots$ & $\ldots$ & $\ldots$ & $\ldots$ & $\ldots$ & 1080 & $\ldots$ & 1104 & 15.3 \\
\hline $4+5 \ldots \ldots \ldots \ldots \ldots \ldots$ & 7.2 & $\ldots$ & $\ldots$ & $\ldots$ & $\ldots$ & $\ldots$ & $\ldots$ & 1290 & $\ldots$ & 1216 & $7.9^{\mathrm{b}}$ \\
\hline
\end{tabular}

Notes.-Aperture integrated optical and IR flux densities of the star-forming knots in J1023+1952 (see Fig. 8 for the knot identifications). Flux densities are uncorrected for extinction. The $\mathrm{H} \alpha$ fluxes include a correction factor of 0.77, derived from our spectroscopy (see Table 2), for $\mathrm{N}$ II emission. For the Spitzer fluxes we applied aperture correction, based on the assumption that the knots are point sources. The correction factors used were $(1.13,1.14,1.20,1.24,1.66)$ at $(3.6,4.8,5.8,8,15 \mu \mathrm{m})$ for the $3.6^{\prime \prime}$ aperture, $(1.16,1.90)$ at $(8,24 \mu \mathrm{m})$ for the $4.9^{\prime \prime}$ aperture, and $(1.07,1.60)$ at $(8,24 \mu \mathrm{m})$ for the $7.2^{\prime \prime}$ aperture. The error of the fluxes is estimated to be about $10 \%$ at all wavelengths due to uncertainties in the background subtraction, and $30 \%$ at $24 \mu \mathrm{m}$ due to the more elaborate image processing that was necessary (see $\S 2.3$ ).

a Aperture radius of $4.9^{\prime \prime}$.

$\mathrm{b}$ The sum of the fluxes of knots 4 and 5 is slightly higher than the integrated flux from both knots because of contamination with the adjacent knot in the $3.6^{\prime \prime}$ aperture (see $\S 3.3 .2$ ). 


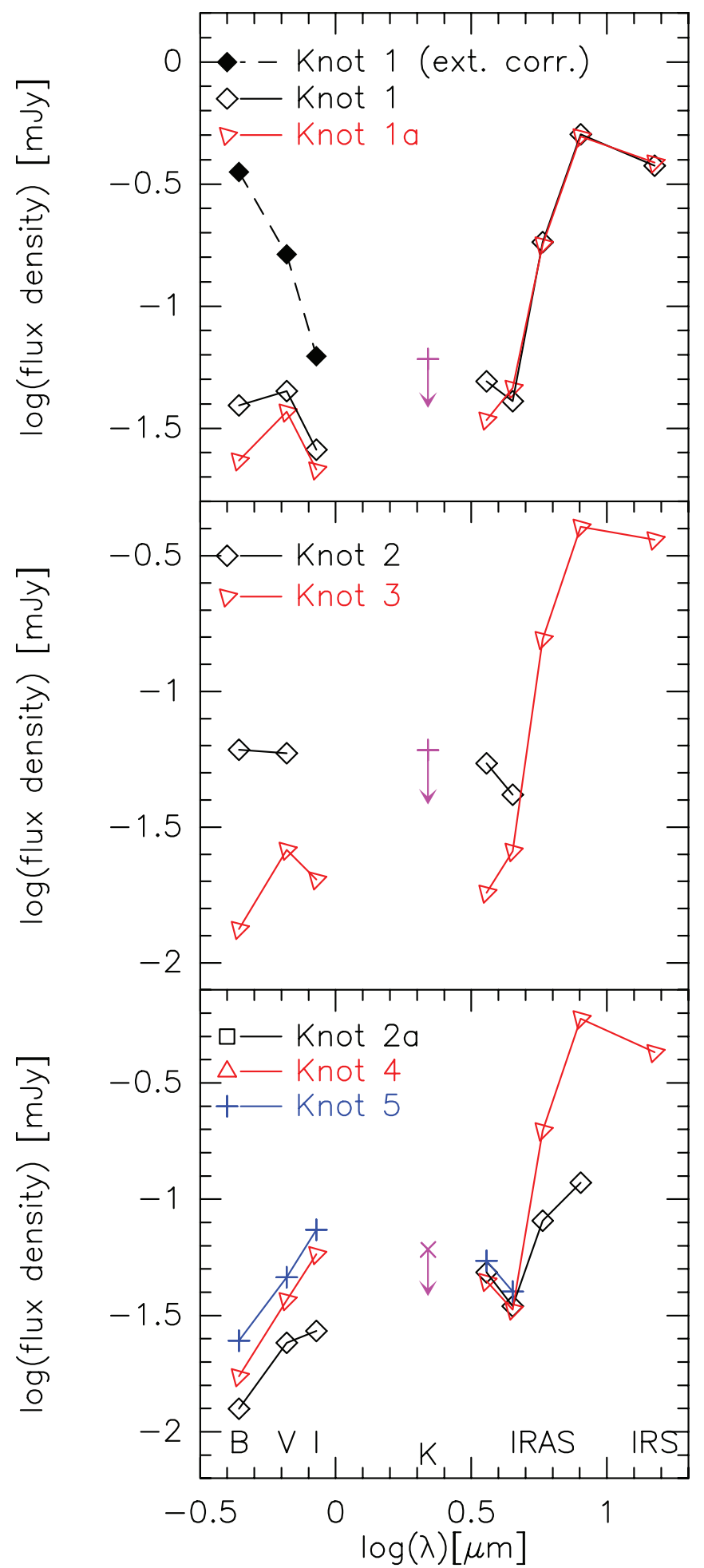

FIG. 9.-Optical+IR SEDs of the SF knots. The fluxes used here are listed in Table 3 and are uncorrected for extinction, except for knot 1 , where we show both the uncorrected (open symbols and solid lines) and the extinction-corrected (filled symbols and dashed line) fluxes, using $A_{\mathrm{H} \alpha}=1.4$ mag as measured and adopting the extinction curve of Draine (2003) to derive the extinction at other wavelengths. The upper limit in the $K$ band is from Mundell et al. (2004) and is valid for all knots.

order to achieve reasonable agreement between the data and simulations and derive for this case a starburst age of 4-5 Myr (both for an instantaneous and a continous burst). Higher values for the extinction than the reference value of $A_{B}=2.4$ mag measured in knot 1 would indicate a younger age than 1-2 Myr. Thus, in spite of the uncertainty in the extinction, we can constrain the ages of these two SF knots to be at most 5 Myr.

3. Knot 2 is the brightest knot in the $B$-band image and has an even bluer color than knots $1,1 \mathrm{a}$, and 3 , suggesting a similarly young age. However, knot 2 shows no $\mathrm{H} \alpha$, indicating that no very young SF is taking place. A possible explanation is the absence of dust locally at knot 2 . This hypothesis is consistent with the absence of long-wavelength emission from small grains or PAH molecules. The comparison of Starburst99 simulations to the extinction-free SED yielded ages of 20-100 Myr (for instantaneous SF) and 50-600 Myr (for a continous burst). These relatively old ages would explain the lack of $\mathrm{H} \alpha$ emission.

4. The other knots (knots 4, 5, and 2a) have redder optical SEDs, either due to a higher extinction or due to an older stellar population. Knots 4 and 5 show $\mathrm{H} \alpha$ emission, whereas from knot 2a no $\mathrm{H} \alpha$ emission could be detected. If the extinction at these knots had values of $A_{B}=3.5-4$ mag, the intrinsic SED would be similar to knot 1 , and we would derive a similarly young SF age. Alternatively, assuming that the extinction has the same value as in knot 1, we derive for knots 4 and 5 ages of 10-100 Myr (continous SF) and 5-6 Myr (instantaneous burst). For knot 2a we derive an age of about $5 \mathrm{Myr}$ for both burst scenarios. If the extinction were lower, the ages of the knots would be higher. In the limiting case of no extinction we derive satisfactory fits for all three knots for a starburst age of $100 \mathrm{Myr}$ (for an instantaneous burst; for a continous burst no good fit could be achieved). This value is certainly an overestimate, at least for knots 4 and 5, because in this case we would not expect any $\mathrm{H} \alpha$ emission.

Interestingly, there are differences in the $\mathrm{H} \alpha$ and dust emission: whereas knot 4 has clear $\mathrm{H} \alpha$ and dust emission, the dust emission (mostly visible in the $8 \mu \mathrm{m}$ map, since the lower spatial resolution of the other wavelengths makes a clear spatial association difficult) at knot 5 is, if present, at most weak compared to its $\mathrm{H} \alpha$. Such drastic variations between the $8 \mu \mathrm{m}$ and $\mathrm{H} \alpha$ emission have been observed occasionally in other intergalactic SF regions as well (e.g., Boquien et al. 2007) and are most likely due to the fact that PAHs can be easily destroyed in a strong UV field.

In summary, we find that the SEDs of all knots, except for knot 2, are consistent with recent SF ( $<10 \mathrm{Myr}$ ). Furthermore, assuming that the extinction does not vary substantially over the SF region, and in particular that nowhere are the values much higher than measured in knot 1, we find tentative evidence for an age gradient across the knots: the oldest knots, with ages most likely between 5 and $20 \mathrm{Myr}$, lie in the south to southeast (4, 5, and $2 \mathrm{a}$ ), while the remaining knots $(1,1 \mathrm{a}$, and 3 ) are very young, at most 2 Myr. Knot 2 represents an exception in this sequence with most likely an older age.

\subsubsection{Comparing Tracers of Star Formation}

The use of the 8 and $24 \mu \mathrm{m}$ emission as a tracer for obscured SF has been studied in numerous galaxies. The $24 \mu \mathrm{m}$ emission shows a tight relation with the emission of the ionized gas (Calzetti et al. 2005, 2007; Pérez-González et al. 2006) which is independent of metallicity if only the $\mathrm{H}$ II regions are taken into account (Relaño et al. 2007). There exists also a relation between the $8 \mu \mathrm{m}$ emission and the ionized gas emission, but it is less tight and more dependent on metallicity (Calzetti et al. 2007).

Figure 10 shows a comparison between the luminosities of the extinction-corrected $\mathrm{H} \alpha, 8$ and $24 \mu \mathrm{m}$, respectively, for the knots where both emissions have been detected, together with a comparison to data from the literature for M81 (Pérez-González et al. 2006), M51 (Calzetti et al. 2005), and, in the case of the $8 \mu \mathrm{m}$ 

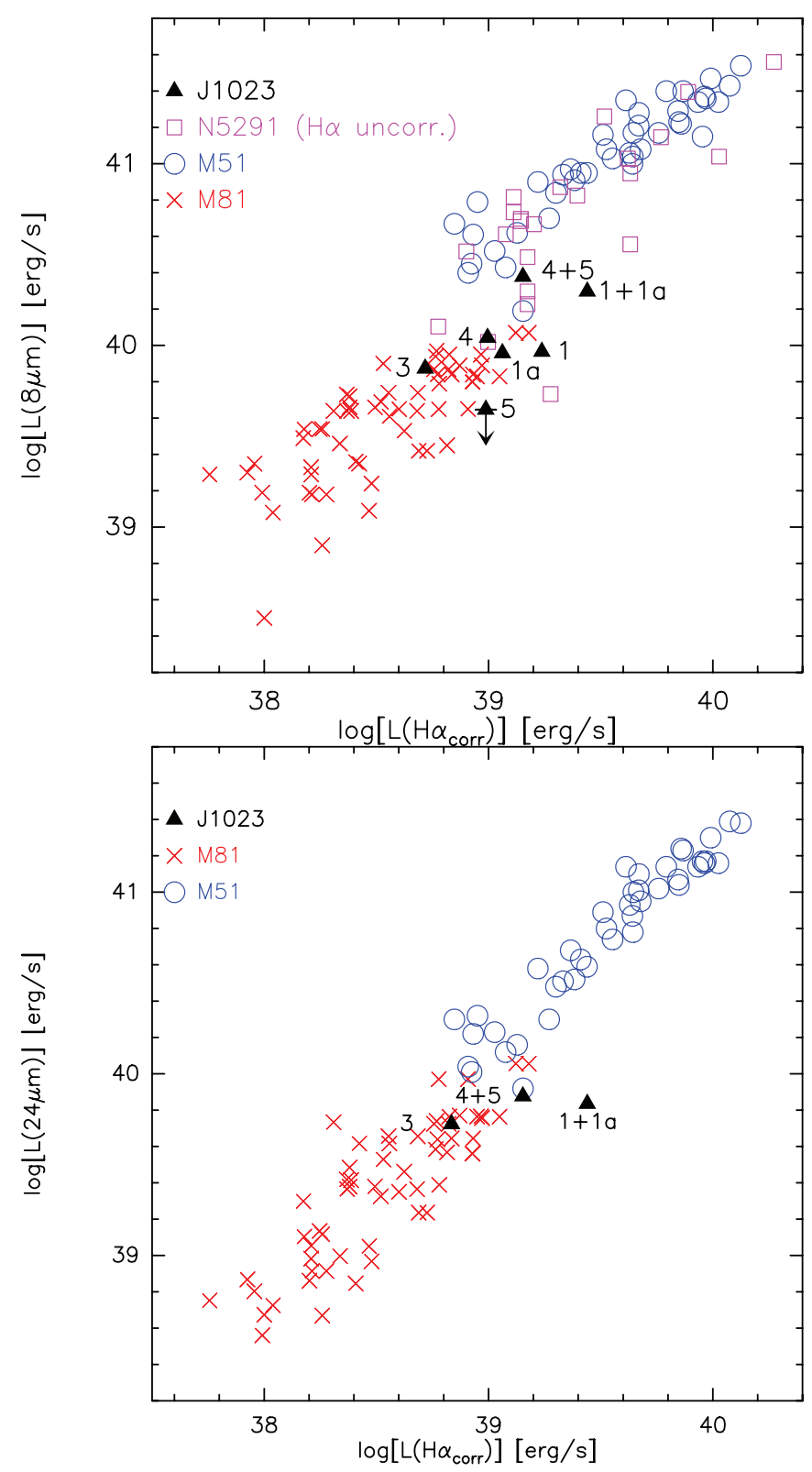

FIG. 10.-Top: $8 \mu \mathrm{m}$ luminosity and the extinction-corrected $\mathrm{H} \alpha$ luminosity (assuming an extinction of $A_{\mathrm{H} \alpha}=1.4 \mathrm{mag}$ ) of the knots $1,1 \mathrm{a}, 3,4$, and 5 , as well as the combined emission of knots $1+1 \mathrm{a}$ and $4+5$ together with data of $\mathrm{H}$ II regions in M51 (Calzetti et al. 2005), M81 (Pérez-Gonzalez et al. 2006), and extragalactic $\mathrm{H}$ II regions in NGC 5291 (Boquien et al. 2007). For the latter the $\mathrm{H} \alpha$ luminosity is uncorrected for extinction. Bottom: The $24 \mu \mathrm{m}$ luminosity and the extinction-corrected $\mathrm{H} \alpha$ luminosity of the knots $1+1 \mathrm{a}$ (combined), knot 3 and knots $4+5$ (combined) together with data of $\mathrm{H}$ II regions in M51 (Calzetti et al. 2005) and M81 (Pérez-Gonzalez et al. 2006).

emission, with the extragalactic $\mathrm{H}$ II regions in the system NGC 5291 (Boquien et al. 2007).

The relation between the $8 \mu \mathrm{m}$ and $\mathrm{H} \alpha$ emission of the four knots (1, 1a, 3, and 4) in J1023+1952 matches those of the $\mathrm{H}$ II regions in M81. We also include the combined emission of knots $1+1 \mathrm{a}$ and knots $4+5$ which were used in the analysis of the $24 \mu \mathrm{m}$. Knots 1+1a show a slight excess of $\mathrm{H} \alpha$ emission, or a lack of $8 \mu \mathrm{m}$ emission, with respect to the data points of M51 and M81.

The $24 \mu \mathrm{m}$ emission of knots 1 and 1a as well as of knots 4 and 5 could not be measured individually, so that the combined emission of both knots is considered whenever a comparison to the $24 \mu \mathrm{m}$ emission is made. The corresponding values are listed in Table 3 . Whereas knots $4+5$ and knot 3 follow very well the correlation between the $24 \mu \mathrm{m}$ and the extinction-corrected $\mathrm{H} \alpha$ luminosity of the $\mathrm{H}$ II regions in M81 and M51, the emission of knots 1+1a shows an excess of $\mathrm{H} \alpha$ emission, or a lack of $24 \mu \mathrm{m}$ emission, with respect to the correlation. The reason for this difference is unclear. The uncertainty in the $24 \mu \mathrm{m}$ correction (for the AGN contamination in the center of NGC 3227) could explain part of this discrepancy. Alternatively, given that the excess of $\mathrm{H} \alpha$ luminosity is relatively small (a factor of $\sim 1.5-2$ ), a slight overestimate of the extinction could also explain the apparent excess.

\section{DISCUSSION}

\subsection{Triggering the Star Formation}

SF in J1023+1952 is restricted to a small region in the southern part. Our observations show that the absence of SF in the rest of the cloud is not due to a lack of molecular gas because abundant $\mathrm{CO}$ has been found over the entire object. Figure 5 shows that the surface density of the molecular gas is relatively uniform over the cloud, with values ranging between 3 and $6 \times 10^{20} \mathrm{~cm}^{-2}$ (Table 1). The column density of the total neutral (atomic and molecular) gas is rather homogeneous as well with values ranging between $\sim 1$ and $3 \times 10^{21} \mathrm{~cm}^{-2}$ (see Table 1). For these values of gas column density, SF activity has routinely been found in TDGs (Braine et al. 2001). The atomic gas surface density peaks in the $\mathrm{SF}$ region (see Fig. 5), but other places along the $\mathrm{H}$ I ridge have similarly high values, so that this high value alone cannot explain why SF is occurring in just one restricted area.

A noticeable difference in the gas properties is the narrower line width both of the $\mathrm{CO}$ and the $\mathrm{H} \mathrm{I}$ in the region where SF takes place in comparison to the rest of the cloud. Thus, SF is only present where the gas is dynamically cold, whereas in the rest of the cloud the higher velocity dispersion of the molecular gas clouds seems to suppress SF.

Our observations thus show that overall gas richness is not a sufficient condition for SF, and that the dynamics of the gas have to be considered as well. Studies by other authors agree with this result. In the dwarf galaxy VCC 2062, most likely an old TDG, SF has also been found only in the dynamically cold gas (Duc et al. 2007).

In a study of SF in tidal tails, Maybhate et al. (2007) found that in order for SF to take place in tidal arms, a high gas surface density $\left(\log N_{\mathrm{H}}>20.6 \mathrm{~cm}^{-2}\right)$ is a necessary but not sufficient condition. In fact, they found several places in the tidal arms of NGC 4038/39 and NGC 3921 where no stellar clusters were found in spite of a high gas surface density. From our study, we would predict that the gas in the locations without SF has a considerably higher velocity width than in the places where SF takes place.

The process of SF can empirically be well described by a Schmidt law for the SFR and a threshold below which SF is inhibited (Kennicutt 1998a). The SFR in the southern region follows well the law found by Kennicutt (1998b) for a large sample of galaxies. Both the gas column density and the SFR per area in this region lie at the lower end of the range of values found in galaxies (Kennicutt 1998b) and thus close to the values where thresholds become important.

There are two kinds of thresholds in gas column density that have to be considered (Elmegreen 2002). A minimal gas column density (of about $6 M_{\odot} \mathrm{pc}^{-2}$ for a typical radiation field and a solar-like metallicity; Elmegreen 2002) is required to provide a high enough pressure to support a cool phase of $\mathrm{H}$ I clouds, necessary as a first step for SF. Apart from this, a critical column density exists below which large clouds cannot form, either due to destructive Coriolis forces (the Toomre criterion; Toomre 
1964) or large-scale shears (Elmegreen 1987, 1991; Hunter et al. 1998).

In $\mathrm{J} 1023+1952$ the gas column density ranges between 10 and $20 M_{\odot} \mathrm{pc}^{-2}$, which is above typical values for the minimum gas column densities. Further and strong evidence showing that the minimum gas column density is exceeded in the entire cloud comes from the fact that the gas is sufficiently dense to support a high molecular gas fraction. We do not find evidence that a threshold based on a critical density can explain the differences in SF in the north and the south of the cloud. The Toomre criterion predicts a critical surface density, $\mu_{\text {crit }}=\sigma \kappa /(\pi G)$, where $\sigma$ is the velocity dispersion within the clouds, $\kappa$ is the epicyclic frequency, and $G$ is the gravitational constant. If we assume that the velocity gradient found in the gas is due to rotation, we derive little variation for $\mu_{\text {crit }}$, with values between 4 and $5 M_{\odot} \mathrm{pc}^{-2}$, within the cloud, and a trend for the lower values being situated in the northern part, so that this criterion cannot explain the absence of SF in the north. A similar conclusion results from the stability criterion based on the shear which yields a critical column density of $\mu_{\text {crit }}=2.5 A \sigma /(\pi G)$ (Elmegreen 1993; Hunter et al. 1998), with $A$ being the Oort constant. The critical gas column densities in this case are slightly higher in the northern part, but all values are below $1 M_{\odot} \mathrm{pc}^{-2}$, so that they cannot explain the lack of SF.

Thus, there must be a different mechanism that inhibits SF in the northern part, e.g., large-scale turbulence which could prevent SF if the motions continuously force the gas to break up to pieces that are smaller than a thermal Jeans mass (Padoan 1995; Elmegreen 2002). Large-scale turbulence would be consistent with the higher velocity width in this region. Alternatively, SF could have been triggered in the cool molecular gas present in the cloud, but only in the southern part, possibly due to gas compression related to the tidal interaction.

\subsection{The Nature and Origin of J1023+1952}

$\mathrm{J} 1023+1952$ is an intriguing object whose origin is a matter of debate. The main scenarios are that J1023+1952 is (1) a classical dwarf galaxy, either involved in the interaction or seen as a chance projection, (2) a small spiral galaxy being accreted and tidally disrupted by the NGC 3226/7 system, (3) made of gas from the disk of NGC 3227 expelled by the impact of a third body, or (4) a potential TDG formed from tidal debris extracted from the gaseous disk of NGC 3227. We discuss these possibilities in turn.

\subsubsection{A Preexisting Dwarf Galaxy?}

One key property that distinguishes "classical" dwarf galaxies (i.e., not formed from recycled gas) from TDGs is their metallicity. Since the latter are made from recycled gas, their metallicities are close to those of the parent galaxies. Therefore, TDGs do not follow the magnitude-luminosity relation found for classical dwarf galaxies; on the contrary, their metallicities lie in a narrow range of $12+\log (\mathrm{O} / \mathrm{H})=8.3-8.6$ (Duc et al. 2000). The total brightness of J1023+1952 of $M_{B} \sim-15.9$ (corrected for an extinction of $A_{B}=2.4$ mag derived from our spectroscopy) would predict a metallicity of $12+\log (\mathrm{O} / \mathrm{H}) \sim 8.0$. This is well below the metallicity estimated from the optical spectra $(\S 3.2)$ showing that J1023+1952 cannot be a "classical" dwarf galaxy. The detection of large quantities of $\mathrm{CO}$ further confirms this high metallicity of the neutral gas. At low metallicities of $12+\log (\mathrm{O} / \mathrm{H}) \sim 8$ or below the detection rate of classical dwarves in $\mathrm{CO}$ is very low (e.g., Taylor et al. 1998), indicating that $\mathrm{CO}$ is no longer a good tracer of the molecular gas content. In J1023+1952, in contrast, we derived a high molecular-to-atomic gas mass ratio of $60 \%$, which is even higher than the range of values found for TDGs (Braine et al.
2001; Lisenfeld et al. 2002) of $\sim 10 \%-50 \%$. Thus, both observations, the high metallicity and the high molecular gas abundance, lead us to discard the hypothesis that $\mathrm{J} 1023+1952$ is a preexisting dwarf galaxy.

\subsubsection{An Infalling, Disrupted Small Spiral Galaxy?}

Deep optical images of the system (see Fig. 11, or Fig. 3 [left] in Mundell et al. 2004) show faint loops of emission around NGC 3227 indicating the presence of stellar stream. This clearly indicates that something is being accreted onto NGC 3227. J1023+1952 is placed at the intersection of two ends of such loops, suggesting that its existence might be causally related to them. A third object (a small spiral galaxy) falling into the system and being disrupted by the gravitational field of NGC $3227 / 6$ could be the origin of the stellar streams. The stellar content in this accreting object is then being disrupted into long tidal streams around the system. The dissipative gaseous component, on the other hand, does not follow the stellar distribution exactly, but is more concentrated at the position where the streams cross so that the gas clouds collide and lose their energy. This could explain the presence of $\mathrm{J} 1023+1952$ at just this position. The collision of the gas clouds would explain furthermore the onset of recent star formation due to the compression of the gas.

The infalling galaxy would however have to be much smaller than NGC 3227 in order to explain the fact that it is disrupted while NGC 3227 is only very little disturbed. Taking, e.g., a factor of 10 as a reasonable mass and luminosity difference, we would expect an object with a brightness of $M_{B} \sim-17 \mathrm{mag}$. For such a small object, the expected metallicity is much lower than observed, only $12+\log (\mathrm{O} / \mathrm{H}) \sim 8$.0. Turning the argument around, we predict, using the luminosity-metallicity relation, from the metallicity of J1023+1952 a luminosity of $M_{B} \sim-19$ mag for the infalling object, which is practically the same as for NGC 3227. Such a large object, if in interaction with the system, would be expected to cause major damage to NGC 3227 as well. Therefore, J1023+1952 cannot be made of material from a third infalling object; it needs to be made of gas from NGC 3227 or NGC 3226.

\subsubsection{Collisionally Expelled Gas from NGC 3227?}

A further alternative is that a small galaxy has passed through the disk of NGC 3227 and has expelled part of the gas from the disk via cloud-cloud collisions, in the same way as gaseous bridges are formed between galaxies involved in head-on collisions.

In this case $\mathrm{J} 1023+1952$ would be made of gas from the disk of NGC 3227 which would explain its high metallicity. The position-velocity diagram along the major axis of NGC 3227 shown in Figure 7 in Mundell et al. (1995) shows that the gas in $\mathrm{J} 1023+1952$ corresponds roughly to the atomic gas that seems to be missing from the central region of NGC 3227, which is consistent with this scenario. However, in a direct collision one would expect the formation of a gaseous bridge between the two objects, as, e.g., observed in the Taffy galaxies (Condon et al. 1993; Braine et al. 2003, 2004), and not only emission at two discrete velocities, as observed in NGC 3227 and $\mathrm{J} 1023+1952$.

Therefore, although at first sight an attractive possibility, $\mathrm{J} 1023+1952$ cannot be made of collisionally expelled gas from the disk of NGC 3227.

\subsubsection{A Potential TDG?}

Alternatively, J1023+1952 could be a TDG, an object formed from tidal debris stripped from the disk of NGC 3227 during the interaction, explaining in this way naturally the high metallicity and molecular gas content. In comparison to other TDGs which 


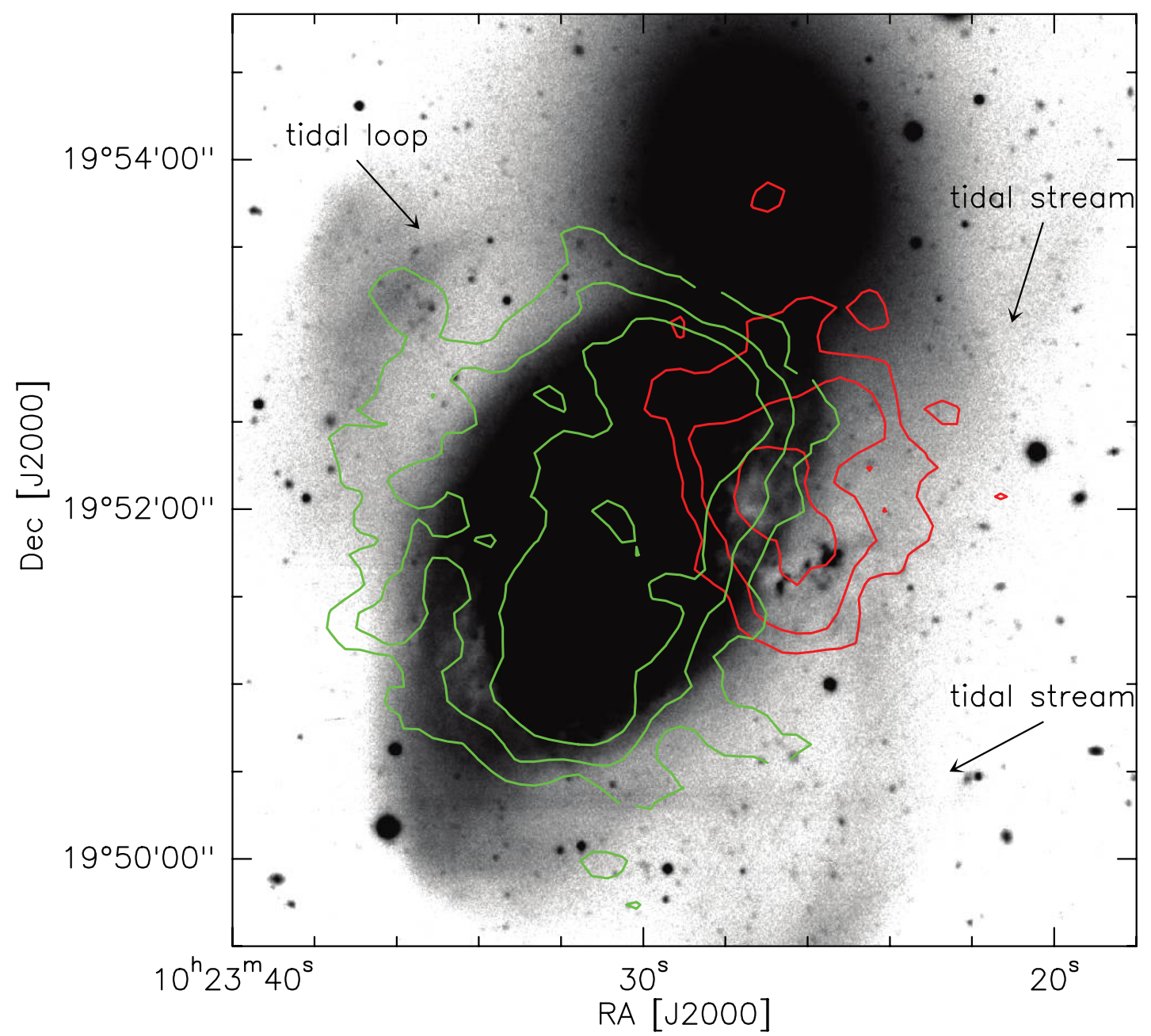

FIG. 11.-Deep B-band image of Arp 94 showing the tidal streams around the object. J1023+1952 is situated at the intersections of two ends of a stream, close (in

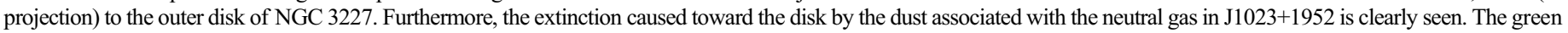

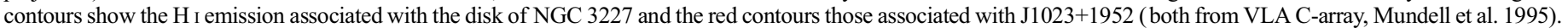

normally lie at the end of tidal tails (e.g., Duc et al. 2004), the position of $\mathrm{J} 1023+1952$ at the base of the northern plume is, however, unusual.

The young age of its stellar populations suggests that it has formed recently and the high metallicity and high molecular gas content imply that it has formed from material coming from the main disk. Possibly it has formed in a second phase of interaction, after a first one which expelled the outer $\mathrm{H}$ i disk and formed the $\mathrm{H}$ I tidal tails. This late and recent formation might explain its unusual position relatively close to NGC 3227 . Evidence for previous removal of atomic gas from the disk of NGC 3227 comes from the relatively strong $\mathrm{CO}$ emission from the southeastern part of the outer disk of NGC 3227 (see Fig. 1). The high ratio of molecular-to-atomic gas surface density $\left[2 N\left(\mathrm{H}_{2}\right) / N\left(\mathrm{H}_{\mathrm{I}}\right)=1.2\right]$ at this position is unusual for outer disks and could be an indication of removal of atomic gas in a previous phase of the interaction.

A suggestive scenario is that the tidal streams, described in $\S 4.2 .2$, are debris from a previous phase of the interaction. They could either have their origin in NGC 3227, representing material from the disk that was expelled in an earlier stage of the interaction, or, in a more speculative but not unrealistic scenario, be related to the formation of NGC 3226. This dwarf elliptical has a lot of unusual features (P. N. Appleton et al., in preparation) including a highly peculiar boxlike outer light distribution, a rapidly rotating nuclear core, and most importantly, long stellar streams emanating from it on deep optical images (see, e.g., Fig. 11). All these factors suggest that either NGC 3226 is itself a merger product, or at the very least, it has accreted material from NGC 3227 . J1023 +1952 is situated at the crossing of two tidal streams. Although it is possible that this spatial coincidence is due to a projection effect, an alternative scenario is that $\mathrm{J} 1023+1952$ has been formed due to the crossing of two tidal streams. In this case, the recycled nature of the gas presumably associated with the tidal streams would explain the high metallicity and high molecular gas content. Our VLA observations (see Fig. 11) indeed show some indications of gas associated with the tidal streams, especially at the eastern side, supporting this possibility. However, deeper VLA observations are required in order to find out whether the stellar streams really contain gas as required in this scenario.

\section{CONCLUSIONS AND SUMMARY}

We presented a comprehensive study of the ISM of J1023+ 1952, based on new millimeter and infrared data, with the goal of determining its nature and origin. The main results and conclusions are

1. ${ }^{12} \mathrm{CO}(1-0)$ and ${ }^{12} \mathrm{CO}(2-1)$ emission was detected for the first time and shown to be extended across the entire $\mathrm{H} \mathrm{I-}$ emitting cloud. The intensity-weighted velocities and the line widths of the $\mathrm{CO}$ and $\mathrm{H}$ I emission agree well. 
2. The optical spectroscopy provided measures of the extinction and metallicity of the brightest SF knot (knot 1). We derived an extinction of $1.4 \mathrm{mag}$ for the $\mathrm{H} \alpha$ line (corresponding to $\left.A_{B}=2.4\right)$ and a metallicity of $12+\log (\mathrm{O} / \mathrm{H})=8.6 \pm 0.2$ showing that the gas is preenriched.

3. We identified seven SF knots in optical and Spitzer images, and presented the results of aperture photometry. The SEDs of these objects were indicative of young star formation (ages between 1 and 10-20 Myr), although they showed surprising differences. We identified a tentative age gradient from the southeast, where the two knots (knots 4 and 5) had the reddest color, to the northwest (knots 1 and 1a), where a very young stellar population was present. One knot (knot 5) had $\mathrm{H} \alpha$ emission with no associated $8 \mu \mathrm{m}$ emission. Knot 2 has the strongest blue emission but showed no $\mathrm{H} \alpha$ and no dust emission in spite of its blue color. A possible reason might be the lack of dust which would lead to an overestimate of the extinction and thus to a too blue color.

4. We studied the properties of the 8 and $24 \mu \mathrm{m}$ emission in comparison with the extinction-corrected $\mathrm{H} \alpha$ emission, in order to investigate the dust properties and the use of these two emissions as SF tracers. Neither the 8-to- $\mathrm{H} \alpha$ ratio nor the 24-to- $\mathrm{H} \alpha$ ratio of most knots showed a significant difference in comparison to $\mathrm{H}$ II regions in M81 and M51. Only for the combined emission of knots 1+1a did we find a slight excess of $\mathrm{H} \alpha$ with respect to the $24 \mu \mathrm{m}$ luminosity.
5. We discussed various possible scenarios for the origin of $\mathrm{J} 1023+1952$. A strong constraint is the high metallicity, which is supported by the abundant $\mathrm{CO}$ emission, much higher than expected from its luminosity if $\mathrm{J} 1023+1952$ were a preexisting dwarf galaxy. Based on this fact, we can exclude a chance superposition of a dwarf galaxy or an infalling, tidally disrupted small galaxy as the origin of J1023+1952. Instead, we conclude that J1023+1952 must have formed from recycled gas from the parent galaxies.

We would like to thank the referee for useful and detailed comments on the draft, as well as Almudena Zurita, Monica Relaño, Beatriz Ruiz, Vicent Martínez, and Cesar Husillos for carrying out the observations at Calar Alto. We would furthermore like to acknowledge the program Calar Alto Academy that allowed these observations to be taken. We are grateful to H. Teplitz (Caltech) for help with IRS peak-up imaging used in this paper and to T. Jarrett (IPAC) for useful discussions. U. L. thanks IPAC for its hospitality during a summer visit where part of this work was done and acknowledges financial support from the research project AYA 2005-07516-C02-01 and ESP 200406870-C02-02 from the Spanish Ministerio de Ciencia y Educación and from the Junta de Anaducía. C. G. M. acknowledges financial support from the Royal Society and the RCUK.

Facilities: CLFST, CAO:2.2m, Spitzer, IRAM:30m
Asplund, M., Grevesse, \& N, Sauval, A. J. 2005, in ASP Conf. Ser. 336, Cosmic Abundances as Records of Stellar Evolution and Nucleosynthesis, ed. T. G. Barnes III \& F. N. Bash (San Francisco: ASP), 25

Barnes, J. E., \& Hernquist, L, 1992, Nature, 360, 715

Boquien, M., et al. 2007, A\&A, 467, 93

Braine, J., \& Combes, F. 1992, A\&A, 264, 433

Braine, J., Davoust, E., Zhu, M., Lisenfeld, U., Motch, C., \& Seaquist, E. R. 2003, A\&A, 408, L13

Braine, J., Duc, P.-A., Lisenfeld, U., Charmandaris, V., Vallejo, O., Leon, S., \& Brinks, E. 2001, A\&A, 378, 51

Braine, J., Lisenfeld, U., Duc, P.-A., Brinks, E., Charmandaris, V., \& Leon, S. 2004, A\&A, 418, 419

Braine, J., Lisenfeld, U., Duc, P.-A., \& Leon, S. 2000, Nature, 403, 867

Brinchmann, J., et al. 1998, ApJ, 499, 112

Calzetti, D., et al. 2005, ApJ, 633, 871 2007, ApJ, 666, 870

Condon, J. J., Helou, G., Sanders, D. B., \& Soifer, B. T. 1993, AJ, 105, 1730

Cowie, L. L., Songaila, A., Hu, E. M., \& Cohen, J. G. 1996, AJ, 112, 839

Denicoló, G., Terlevich, R., \& Terlevich, E. 2002, MNRAS, 330, 69

Draine, B. T. 2003, ARA\&A, 41, 241

Duc, P.-A., Bournaud, F., \& Masset, F. 2004, A\&A, 427, 803

Duc, P.-A., Braine, J., Lisenfeld, U., Brinks, E., \& Boquien, M. 2007, A\&A, 475,187

Duc, P.-A., Brinks, E., Springel, V., Pichardo, B., Weilbacher, P., \& Mirabel, I. F. 2000, AJ, 120, 1238

Edmunds, M. G., \& Pagel, B. E. J. 1984, MNRAS, 211, 507

Elmegreen, B. G. 1987, ApJ, 312, 626

1991, ApJ, 378, 139

1993, in Star Formation, Galaxies, and the Interstellar Medium, ed. J.

Franco, F. Ferrini, \& G. Tenorio-Tagle (Cambridge: Cambridge Univ. Press), 377

- 2002, ApJ, 577, 206

Gerhard, O., et al. 2002, ApJ, 580, L121

Haynes, P., Giovanelli, R., \& Chincarini, G. L. 1984, ARA\&A, 22, 445

Hibbard, J. E., \& van Gorkom, J. H. 1996, AJ, 111, 655

Hunter, D. H., Elmegreen, B. G., \& Baker, A. L. 1998, ApJ, 493, 595
Kennicutt, R. C., Jr. 1998a, ApJ, 498, 541 1998b, ARA\&A, 36, 189

Leitherer, C., et al. 1999, ApJS, 123, 3

Lisenfeld, U., Braine, J., Duc, P.-A., Leon, S., Charmandaris, V., \& Brinks, E. 2002, A\&A, 394, 823

Madau, P., Ferguson, H. C., Dickinson, M. E., Giavalisco, M. Steidel, C. C., \& Fruchter, A. 1996, MNRAS, 283, 1388

Maybhate, A., Masiero, J., Hibbard, J. E., Charlton, J. C., Palma, C., Knierman, K. A., \& English, J. 2007, MNRAS, 381, 59

Mihos, J. C. 2001, ApJ, 550, 94

Mundell, C. G., James, P. A., Loiseau, N., Schinnerer, E., \& Forbes, D. 2004, ApJ, 614, 648

Mundell, C. G., Pedlar, A., Axon, D. J., Meaburn, J., \& Unger, S. W. 1995, MNRAS, 277, 641

Nishiyama, K., Nakai, N., \& Kuno, N. 2001, PASJ, 53, 757

Osterbrock, D. E. 1989, Astrophysics of Gaseous Nebulae and Active Galaxies (Mill Valley: University Science)

Padoan, P. 1995, MNRAS, 277, 377

Pagel, B. E. K., \& Edmunds, M. G., Blackwell, D. E., Chun, M. S., \& Smith, G. 1979, MNRAS, 189, 95

Pahre, Michael, A., Ashby, M. L. N., Fazio, G. G., \& Willner, S. P. 2004, ApJS, 154,229

Pérez-González, P. G., et al. 2006, ApJ, 648, 987

Relaño, M., Lisenfeld, U., Pérez-González, P. G., Vílchez, J. M., \& Battaner, E. 2007, ApJ, 667, L141

Rubin, V, C., \& Ford, W. K. 1968, ApJ, 154, 431

Ryan-Weber, E. V., et al. 2004, AJ, 127, 1431

Sakai, S., Kennicutt, R. C., Jr., van der Hulst, J. M., \& Moss, C. 2002, ApJ, 578,842

Schinnerer, E., Eckart, A., \& Tacconi, L. J. 2000, ApJ, 533, 826

Taylor, C. L., Kobulnicky, H., \& Skillman, E. D. 1998, AJ, 116, 2746

Thomas, D., Maraston, C., Bender, R., \& de Oliveira, C. M. 2005, ApJ, 621, 673

Toomre, A. 1964, ApJ, 139, 1217

Toomre, A., \& Toomre, J. 1972, ApJ, 178, 623

Treu, T., Ellis, R. S., Liao, T. X., \& van Dokkum, P. G. 2005, ApJ, 622, L5

Tully, R. B. 1988, Nearby Galaxies Catalog (Cambridge: Cambridge Univ. Press) 